\title{
OPTIMAL WAVE PROPAGATION-BASED NONDESTRUCTIVE TEST DESIGN FOR QUANTITATIVE DAMAGE CHARACTERIZATION
}

\author{
by \\ Zhanpeng Hao \\ BS in Civil Engineering, Guangdong University of Technology, \\ China, 2012 \\ Submitted to the Graduate Faculty of \\ the Swanson School of Engineering in partial fulfillment \\ of the requirements for the degree of \\ Master of Science
}

University of Pittsburgh

2014 


\section{UNIVERSITY OF PITTSBURGH \\ SWANSON SCHOOL OF ENGINEERING}

This thesis was presented

by

Zhanpeng Hao

It was defended on

March 31st, 2014

and approved by

John C. Brigham, PhD, Assistant Professor, Department of Civil and Environmental Engineering and Bioengineering

Jeen-Shang Lin, ScD, Associate Professor, Department of Civil and Environmental Engineering

Qiang Yu, PhD, Assistant Professor, Department of Civil and Environmental Engineering

Thesis Advisor: John C. Brigham, PhD, Assistant Professor, Department of Civil and Environmental Engineering and Bioengineering 
Copyright (C) by Zhanpeng Hao 2014 


\title{
OPTIMAL WAVE PROPAGATION-BASED NONDESTRUCTIVE TEST DESIGN FOR QUANTITATIVE DAMAGE CHARACTERIZATION
}

\author{
Zhanpeng Hao, M.S. \\ University of Pittsburgh, 2014
}

Nondestructive testing (NDT) has been widely used for damage identification and inverse characterization of material properties in several fields of science and engineering, from structural engineering to medicine. However, there are several common challenges inherent in the evaluation of structures and systems, including the potential for excessive computational expense and ill-posedness of the inverse problem. Numerical methods, such as the finite element method, provide substantial benefits in terms of solution capabilities, but the analysis for NDT applications in realistic structures often requires substantial computational time and power. Furthermore, limitations on the quantity and quality of measurement data can cause the evaluation problem to require even more computational effort and/or lead to solution non-uniqueness or nonexistence.

The present work introduces a general approach to optimal wave propagation-based NDT design for damage characterization applications. More specifically, the objective of this work is to improve the accuracy and efficiency of the damage characterization process by optimizing the parameters of the NDT, such as the locations of sensors and actuators. The NDT design approach developed is based on maximizing the sensitivity of the NDT response measurements to changes in the material properties to be determined by the evaluation, while simultaneously minimizing the redundancy of response measurements. Two simulated case studies are presented to evaluate the performance of the optimal wave propagation-based NDT design approach. Both examples consisted of thin plate structures with a damage field that was represented by changes in the Young's modulus distribution throughout the 
structure. In order to provide practical relevance, the NDT method considered was based on commonly used ultrasonic testing with piezoelectric sensors and actuators. The optimal NDT designs corresponding to maximized sensitivity and minimized response redundancy are shown to provide substantially improved evaluation solution efficiency and accuracy for quantitative damage characterization in comparison to standard approaches. 


\section{TABLE OF CONTENTS}

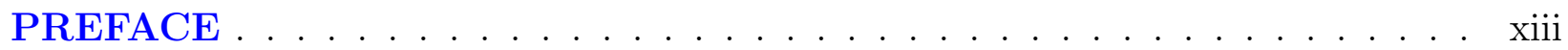

1.0 INTRODUCTION AND LITERATURE REVIEW . . . . . . . . . . . . 1

1.1 Introduction . . . . . . . . . . . . . . . . . 1

1.2 Nondestructive Testing . . . . . . . . . . . . . . . . . . . . 3

1.3 Ultrasonic Testing . . . . . . . . . . . . . . . . . . . . . . . 4

1.3.1 Piezoelectric Transducers . . . . . . . . . . . . . . . . . . 4

1.3 .2 Implementations . . . . . . . . . . . . . . . . . . . 5

1.3.3 Simulation of Wave Propagation . . . . . . . . . . . . 9

1.3.4 Inverse Problems in Structural Dynamics _. . . . . . . . . . 10

1.3.4.1 Inverse Problem Overview: . . . . . . . . . . . . . . 10

1.3.4.2 Optimization Approaches: . . . . . . . . . . . . . . . . . 13

1.4 Optimal Nondestructive Test Design _ . . . . . . . . . . . . . 17

2.0 FORWARD PROBLEM SIMULATION USING THE FINITE ELEMENT METHOD . . . . . . . . . . . . . . . . . . . . . 20

2.1 Wave Propagation in Elastic Solids . . . . . . . . . . . . . . . 20

2.2 Finite Element Formulation for Solid Dynamics . . . . . . . . . . . . 21

2.3 Numerical Time Integration . . . . . . . . . . . . . . . . . . 25

2.3.1 Explicit Time Integration $\ldots \ldots \ldots \ldots$

2.3 .2 Stable Time Increment . . . . . . . . . . . . . . . . . . 27

2.4 Verification of the Forward Model . . . . . . . . . . . . . . . . 28

3.0 INVERSE CHARACTERIZATION SOLUTION STRATEGY . . . . . 32

3.1 Inverse Problem Formulation . . . . . . . . . . . . . . . . . 32 
3.2 Surrogate-Model Accelerated Random Search Algorithm . . . . . . . . . 33

3.2 .1 Random Search Algorithm . . . . . . . . . . . . . . . . . 34

3.2 .2 Surrogate-Model Method . . . . . . . . . . . . . . . . . . 34

3.2 .3 SMARS algorithm . . . . . . . . . . . . . . . 35

4.0 OPTIMIZATION OF NONDESTRUCTIVE TESTING . . . . . . . 38

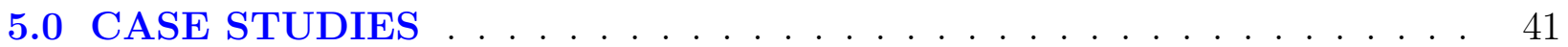

5.1 Implementation of NDT Design and Inverse Characterization Strategies . . 42

5.1.1 Optimal Nondestructive Test Design . . . . . . . . . . . . . . 44

5.1.2 Nondestructive Evaluation/Inverse Characterization . . . . . . . 45

5.2 Example 1 - Square Plate . . . . . . . . . . . . . . . . . . 46

5.3 Example 2 - L Shaped Plate . . . . . . . . . . . . . . . 56

6.0 CONCLUSIONS AND FUTURE DIRECTIONS . . . . . . . . . . . 67

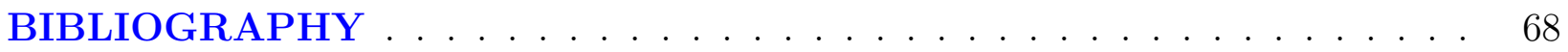




\section{LIST OF TABLES}

5.1 Values of the sensitivity objective function (Eqn. (5.3))and the redundancy objective function (Eqn. (5.4)) for Optimal NDT and Control NDT. . . . . .

5.2 The solution and the relative $L_{2}$-Error and the relative $L_{\infty}$-Error of the Young's modulus distribution with respect to the expected results estimated by the inverse characterization process in the Optimal NDT design and the Control NDT design for Example 1 - Square Plate with damage location (1).

5.3 The solution and the relative $L_{2}$-Error and the relative $L_{\infty}$-Error of the Young's modulus distribution with respect to the expected results estimated by the inverse characterization process in the Optimal NDT design and the Control NDT design for Example 1 - Square Plate with damage location (2).

5.4 The solution and the relative $L_{2}$-Error and the relative $L_{\infty}$-Error of the Young's modulus distribution with respect to the expected results estimated by the inverse characterization process in the Optimal NDT design and the Control NDT design for Example 1 - Square Plate with damage location (3).

5.5 The solution and the relative $L_{2}$-Error and the relative $L_{\infty}$-Error of the $\mathrm{Y}$ oung's modulus distribution with respect to the expected results estimated by the inverse characterization process in the Optimal NDT design and the Control NDT design for Example 1 - Square Plate with one damage location (4).

5.6 Mean and standard deviation of the relative $L_{2}$-Error and the relative $L_{\infty^{-}}$ Error of the Young's modulus distribution for four sets of damage parameters. 
5.7 Values of the sensitivity objective function (Eqn. (5.3))and the redundancy objective function (Eqn. (5.4)) for Optimal NDT and Control NDT. . . . . .

5.8 The solution and the relative $L_{2}$-Error and the relative $L_{\infty}$-Error of the Young's modulus distribution with respect to the expected results estimated by the inverse characterization process in the Optimal NDT design and the Control NDT design for Example 2 - L shape plate with damage parameters (1).

5.9 The solution and the relative $L_{2}$-Error and the relative $L_{\infty}$-Error of the $\mathrm{Y}$ oung's modulus distribution with respect to the expected results estimated by the inverse characterization process in the Optimal NDT design and the Control NDT design for Example 2 - L shape Plate with damage parameters (2).

5.10 The solution and the relative $L_{2}$-Error and the relative $L_{\infty}$-Error of the $\mathrm{Y}$ oung's modulus distribution with respect to the expected results estimated by the inverse characterization process in the Optimal NDT design and the Control NDT design for Example 2 - L shape Plate with damage parameters (3).

5.11 The solution and the relative $L_{2}$-Error and the relative $L_{\infty}$-Error of the Young's modulus distribution with respect to the expected results estimated by the inverse characterization process in the Optimal NDT design and the Control NDT design for Example 2 - L shape Plate with one damage parameters (4).

5.12 Mean and standard deviation of the relative $L_{2}$-Error and the relative $L_{\infty^{-}}$ Error of the Young's modulus distribution for four damage locations. . . . . . 


\section{LIST OF FIGURES}

1.1 Schematic of Pulse-Echo approach for Ultrasonic Testing [1]. . . . . . . . . . 6

1.2 Schematic of Pitch-Catch approach for Ultrasonic Testing [1] . . . . . . . . 7

1.3 Schematic of Through-Transmission approach for Ultrasonic Testing [1] . . . 7

1.4 Schematic of the process of Ultrasonic Testing for nondestructive evaluation [2]. 8

1.5 Comparison of a tone burst signal received from a finite element model using approximate excitation, as well as analytical and experimental results [3] . . . 10

1.6 Schematic of inverse problems in structural dynamics. . . . . . . . . . . . 11

1.7 Flowchart of a conventional Genetic Algorithm (GA) . . . . . . . . . 15

2.1 Time domain transient signal for (a) 5 cycles of a $50 \mathrm{kHz}$ sine wave and (b) 5 cycles of a tone burst with $50 \mathrm{kHz}$ center frequency. . . . . . . . . . . 21

2.2 Schematic for the verification example. . . . . . . . . . . . . . . 29

2.3 Comparison of the vertical displacement between ABAQUS (red solid line) and FEniCS (blue dash line) (a) at coordinate $(0.25 \mathrm{~m}, 0.25 \mathrm{~m})$ and (b) at coordinate

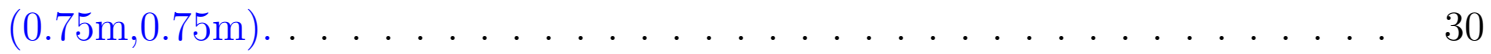

3.1 Flowchart of the surrogate-model accelerated random search algorithm, including the global search random search algorithm component and the local search surrogate-model method component. . . . . . . . . . . . . . . .

5.1 Schematics of the excitation simulated by (a) 12 self-equilibrating forces and (b) the time signal of a 3-cycle tone burst with a center frequency of $50 \mathrm{kHz}$. 
5.2 Schematics of (a) the uniformly distributed sensor locations for the Control NDT design and (b) the sensor locations obtained by the Optimal NDT design approach, where "+" refers to the horizontal sensor orientation and " $\times$ " refers to the vertical sensor orientation, for Example 1 - Square Plate. . . . . . . . 48

5.3 Normalized vertical strain signal at location, $X_{S}=0.75 \mathrm{~m}, Y_{S}=0.75 \mathrm{~m}$, with healthy Young's modulus distribution and Young's modulus reduction located at $\epsilon_{1}^{x}=0.65 m, \epsilon_{1}^{y}=0.65 m$, where red solid line refers to time signal with Young's modulus reduction and blue dash line refers to time signal with healthy

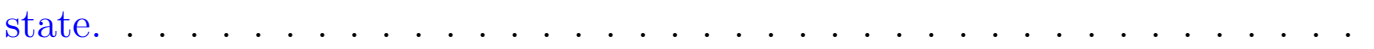

5.4 Normalized vertical strain signal at location, $X_{S}=0.75 \mathrm{~m}, Y_{S}=0.75 \mathrm{~m}$, with healthy Young's modulus distribution before time shift elimination and after time shift elimination where red solid line refers to signal after time shift elimination and blue dash line refers to raw time signal. . . . . . . . . . .

5.5 Spatial Young's modulus distribution of (a) Target (b) inverse characterization by Optimal NDT design (c) inverse characterization by Control NDT design for Example 1 - Square Plate with damage location (1) . . . . . . . . . . 51

5.6 Spatial Young's modulus distribution of (a) Target (b) inverse characterization by Optimal NDT design (c) inverse characterization by Control NDT design for Example 1 - Square Plate with damage location (2) . . . . . . . . . .

5.7 Spatial Young's modulus distribution of (a) Target (b) inverse characterization by Optimal NDT design (c) inverse characterization by Control NDT design for Example 1 - Square Plate with damage location (3) . . . . . . . . . .

5.8 Spatial Young's modulus distribution of (a) Target (b) inverse characterization by Optimal NDT design (c) inverse characterization by Control NDT design for Example 1 - Square Plate with damage location (4) . . . . . . . . . . .

5.9 Schematic of Example 2 - L Shaped Plate and the potential actuator locations for the Optimal NDT design process. . . . . . . . . . . . . . . 56 
5.10 Schematic of (a) the regularly distributed sensor and actuator locations for the Control design and (b) the sensor and actuator locations obtained for the Optimal NDT design, where " + " refers to the horizontal sensor orientation and " $x$ " refers to the vertical sensor orientation, for Example 2 - L Shaped Plate. . . . . . . . . . . . . . . . . . .

5.11 Spatial Young's modulus distribution of (a) Target (b) inverse characterization by Optimal NDT design (c) inverse characterization by Control NDT design for Example 2 - L shape Plate with damage parameters $(1) \ldots . . . .$.

5.12 Spatial Young's modulus distribution of (a) Target (b) inverse characterization by Optimal NDT design (c) inverse characterization by Control NDT design for Example 2 - L shape Plate with damage parameters (2). . . . . . . . . .

5.13 Spatial Young's modulus distribution of (a) Target (b) inverse characterization by Optimal NDT design (c) inverse characterization by Control NDT design for Example 2 - L shape Plate with damage parameters $(3) \ldots$. . . . . . . .

5.14 Spatial Young's modulus distribution of (a) Target (b) inverse characterization by Optimal NDT design (c) inverse characterization by Control NDT design for Example 2 - L shape Plate with damage parameters (4). . . . . . . . . . . 


\section{PREFACE}

I would like to first express my deepest gratitude to my advisor and chair of my graduate

committee, Professor John C. Brigham, for his patience, for being inspirational, and for teaching me the importance of using curiosity as the driving force behind research. Without his unending faith in my potential and his tireless guidance, none of my success could have been possible.

I would like to thank Professor Jeen-Shang Lin, Professor Qiang Yu for serving on my graduate committee and for their support throughout my studies.

I also would like to thank my colleagues in Professor John C. Brigham's group, Bahram, Chenxi, Jing, Mengyu, Mohammad, Oliver, Pei, Shuang, and Weijie, for their help and encouragement during my MS study.

Last, but certainly not least, I would like to thank my family for their love and support, without which I could not have accomplished anything. 


\subsection{INTRODUCTION AND LITERATURE REVIEW}

\subsection{INTRODUCTION}

There has been a considerable amount of work on a variety of computational inverse methods for damage and/or material characterization in various engineering fields ranging from civil, mechanical, and aerospace engineering to biology and medicine $[4,5,6,7,8]$. The basic idea of inverse characterization is that the responses of the system (e.g., forward model outputs, such as displacement, strain, and velocity) from various stimuli carry information about system features that are typically considered known a priori for a forward problem. For example, a structure's responses are functions of the material properties (e.g., mass, stiffness, and damping) of the system, regardless of whether it may be rigid as concrete or a softer material such as tissue. Consequently, any changes in material properties will produce changes in the measurable responses, which could then be used in turn to predict these unknown system properties inversely. Nondestructive testing (NDT) has been employed to excite and measure system responses in several fields, especially solids and structures. Based on system responses from NDT, changes in material properties, caused by damage or otherwise, can be inversely characterized with a variety of methods. A classification for damage characterization methods was defined in [9] as four levels:

Level 1 Determination if damage is present in the structure.

Level 2 Estimation of the geometric location of the damage.

Level 3 Quantification of the severity of the damage.

Level 4 Prediction of the service life of the system. 
Generally, the inverse problems for damage characterization in structures refer to Level 2 or Level 3, which includes the estimation of damage parameters, such as location, breadth, length, and depth. In the present work, both the severity and location parameters will be considered in the damage characterization problem.

One of the typical computational approaches to solve these types of inverse problems is based on updating structural model parameters (e.g., stiffness, mass, and damping) through an iterative procedure. Given measurements from some type of NDT performed on the structure, a computational model is created that predicts the response of the structure from this NDT with respect to an estimate of the unknown parameters. An optimization problem is then created to identify the parameter values that minimize the difference between the responses predicted by the numerical modeling and the measured data from an NDT. There are a wide variety of applications utilizing computational inverse solution methods such as this based on different parameters or material properties, different representations of system responses, and different types of NDT [10, 6, 7, 11, 4]. However, several common procedures are followed. Firstly, the unknown properties of the system need to be defined and parameterized. Secondly, an objective function, which represents the difference between the responses predicted by the computational model and the measured data from the NDT, is defined to be minimized. In addition, boundary conditions, such as the admissible range of the unknown properties, needs to be defined. Lastly, numerical algorithms are employed to perform the optimization to estimate the unknown material properties.

Several issues are present when solving inverse problems for damage and/or material characterization and have been discussed in many previous studies $[12,13]$. One of the important issues to consider is the ill-posedness common to inverse problems. This can lead to non-existence of solutions, non-uniqueness of solutions, and solution instability. Related to these issues is the limited quantity and quality of measurements in many practical applications. Many frameworks that have been developed and shown to work well in certain case studies actually struggle when measurement restrictions exist in practical testing. Alternatively, an approach to optimize the design of NDT by selecting appropriate controllable features, such as actuator location, sensor orientation, location, and frequency, could 
go a long way in relieving some of these issues and improve the solvability of these inverse problems regardless of the specifics of the solution procedure.

\subsection{NONDESTRUCTIVE TESTING}

Nondestructive testing has been developed and used for decades in many fields of engineering. Compared to destructive testing, NDT provides measurement information that can be used for evaluation of the current condition of test objects without changing or destroying their current state and serviceability in any way. As one of the fastest growing technologies over the past 25 years, a large number of different NDT methods have been developed and have advantages suitable for different applications [14]. NDT has been be used in conjunction with inverse solution procedures to determine the presence of damage in structures, as well as estimate the severity and geometric location, leading to the prediction of future performance and estimation of a structure's remaining life span. An overview of some major NDT methods are as follows:

Ultrasonic testing (UT) : Transducer excites the test object with high frequency pulses that propagate through the object. Any reflections from surfaces or discontinuities are then collected by the same transducer or other sensors. This method is suitable for almost all structures and materials and has the ability to also detect small sized defects. Acoustic emission testing (AE) : When the discontinuities develop a stress wave is generated and travels through the structures. This method is often applicable to health monitoring of structural components like bolts and weld connections subjected to loading.

Thermal infrared testing (TIR) : Use thermal detectors such as IR cameras to inspect the temperature distribution of structure's surface. The defects in structures where a change in temperature is related to the thermal conductivity distribution can then be identified. 
The case studies presented in the current work are based upon inverse characterization of properties utilizing UT, which is elaborated upon further in the following.

\subsection{ULTRASONIC TESTING}

Ultrasonic testing has become one of the most important NDT techniques in the fields of civil, mechanical, and aerospace engineering. The potential of UT for nondestructive evaluation (NDE) and structure health monitoring (SHM) has been recognized and discussed for as far back as the 1960s $[15,16,17]$. In UT, an ultrasonic wave, which has a high frequency (i.e., larger than $20 \mathrm{kHz}$ ), is induced by applying a pulse signal (typically a modulated sinusoidal tone burst of some limited number of cycles) through an actuator. The propagation of ultrasonic waves through the test objects or structures is significantly affected by the object's geometry and material properties. In addition, structural discontinuities, which could be damage such as cracks or delamination, or structural boundaries, scatter ultrasonic waves in all direction. Furthermore, changes in material properties affect the features of the wave propagation, such as wave speed, magnitude, etc. Sensors, such as traditional piezoelectric transducers can be employed to collect these responses from the objects or structures being tested.

\subsubsection{Piezoelectric Transducers}

The transducer is a critical component of UT. Piezoelectric transducers (PZT), which are relatively cheap and readily available in small sizes, are the most common transducers used in practice. A PZT is either bonded or embedded into the structure under consideration. The basic functionality of a PZT is that it transforms the mechanical deformation to an electrical signal that can be recorded. The constitutive equation of a PZT can be expressed as:

$$
\epsilon=\frac{\sigma}{E}+\phi \delta
$$


and

$$
D=\alpha \delta+\phi \sigma
$$

Where

$\epsilon=$ mechanical strain

$\sigma=$ mechanical stress

$E=$ Young's Modulus

$\phi=$ piezoelectric strain coefficient

$\delta=$ electric field

$D=$ electrical displacement (charge density)

$\alpha=$ dielectric constant

Utilizing the properties of the PZT transformation between the mechanical deformation and electrical signal, a PZT can be used as both an actuator and a sensor. The capability of a PZT to convert energy between the electrical and mechanical fields can be represented by the coupling coefficient $(k)$ as:

$$
k=\sqrt{\frac{E}{\alpha}} \phi
$$

PZT with larger coupling coefficients are preferred in practice because of their higher efficiency to convert electrical energy into mechanical energy or convert mechanical energy to electrical energy.

\subsubsection{Implementations}

Generally, there are three different approaches to UT, which could be employed individually or collectively: pulse-echo, pitch-catch, and through-transmission [1]. In the pulse-echo approach, PZT or actuator-sensor pairs are used as actuators to excite the structure with a restricted bandwidth pulse, while also being used as sensors to receive the echoes of these pulses reflected by the material discontinuities (e.g., cracks, delaminations, and/or voids) or the structural boundaries. The signals are then digitized and processed, and with a controlled center actuation frequency and known wave speed for the test object, the signals indicating damage can be extracted by applying appropriate filters. A schematic of the pulse-echo approach is shown in Figure 1.1. In the pitch-catch approach a pulse signal sent 


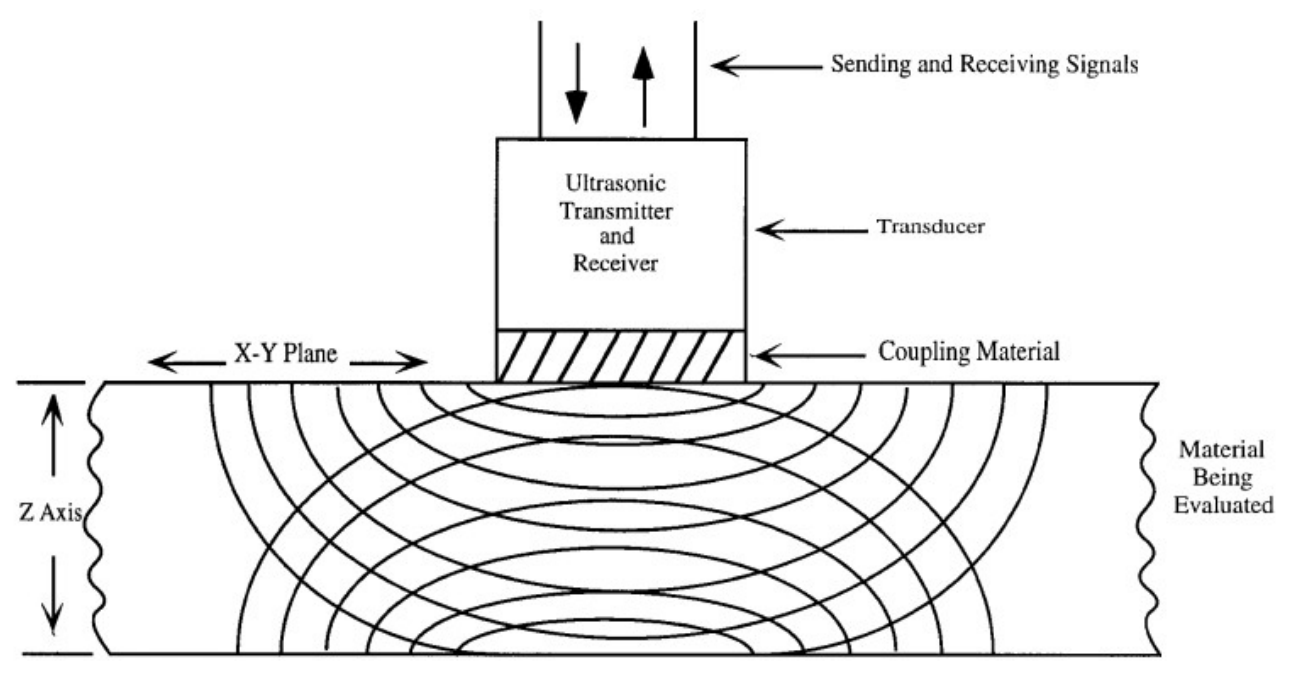

Figure 1.1: Schematic of Pulse-Echo approach for Ultrasonic Testing [1].

by an actuator propagates at an angle to the surface of the structure and is received by a sensor placed at some distance away from the actuator. By analyzing the various features of the collected signal, such as amplitude, transit time, and frequency response, characteristics of damage, including depths, breadth, and location can be determined. A schematic of the pitch-catch approach is shown in Figure 1.2. In the through-transmission approach the actuator and sensor are placed on opposite sides of the test object. This approach is particularly suitable for NDE of multilayered structures or composite materials. A schematic of the pulse-echo approach is shown in Figure 1.3.

All three approaches are well-established in NDE and SHM industries and there have been a large amount of studies on developing these UT methodologies [18, 19, 20, 21, 22, 23]. In every UT approach certain essential steps are followed regardless of the type of test object. The signal collected by the transducers is used as input for some signal-processing algorithms to prepare for further processing and/or extract key features. Based on these signals and/or features, pattern recognition techniques and/or computational inverse solution methods can be employed to determine the types and severity of damage. A schematic of a UT-NDE process is illustrated in Figure 1.4 [2]. 


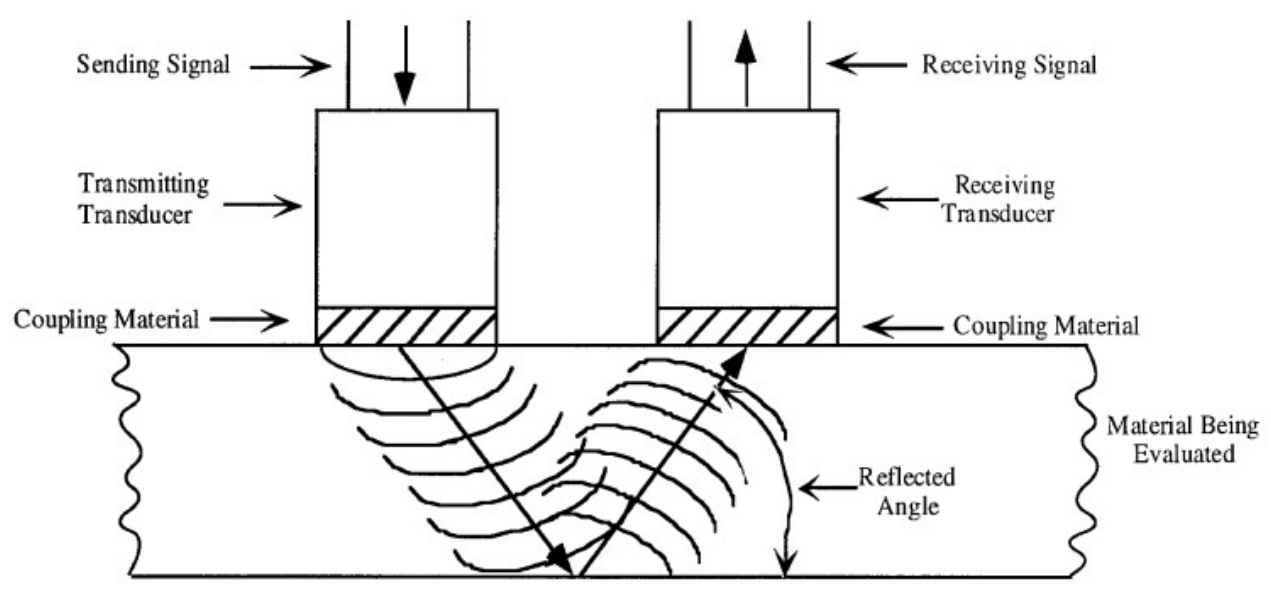

Figure 1.2: Schematic of Pitch-Catch approach for Ultrasonic Testing [1].

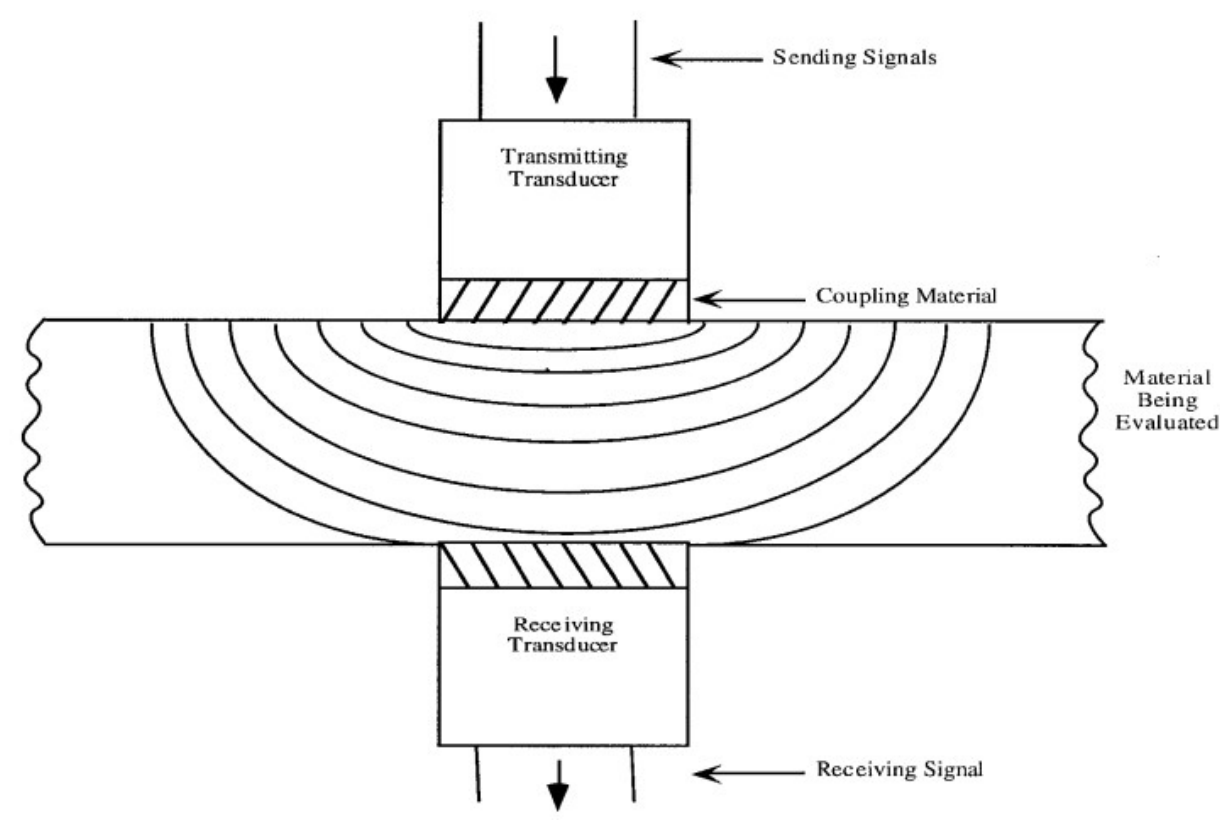

Figure 1.3: Schematic of Through-Transmission approach for Ultrasonic Testing [1]. 


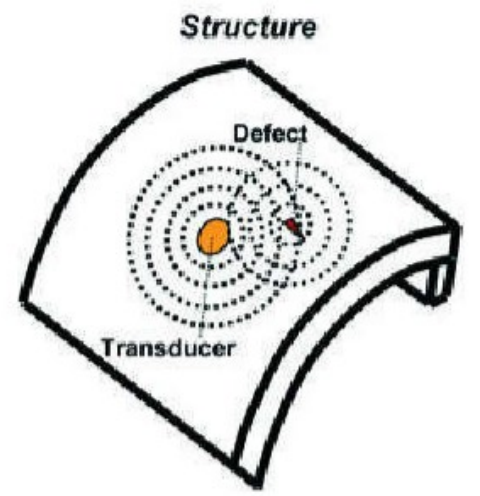

Pattern recognition

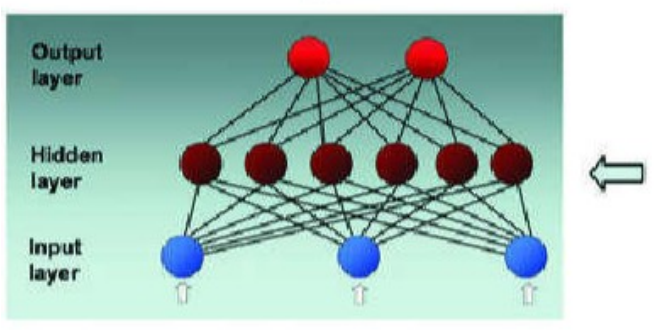

Signal

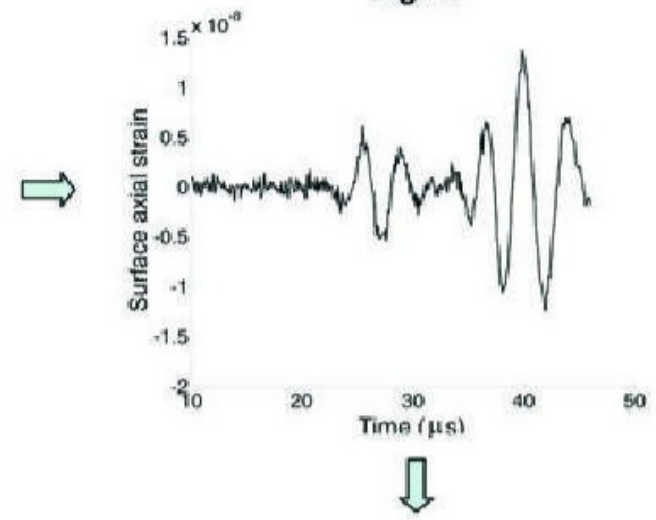

Feature extraction

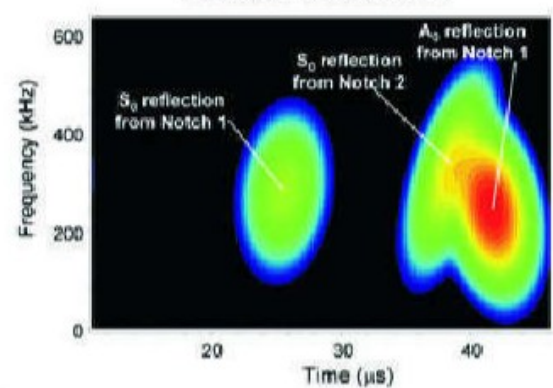

Figure 1.4: Schematic of the process of Ultrasonic Testing for nondestructive evaluation [2]. 


\subsubsection{Simulation of Wave Propagation}

Level 2 and Level 3 NDE damage characterization, which are the determination of the damage location and quantification of the damage severity, can be particularly difficult to achieve based on limited experimental data and using pattern recognition techniques. In such cases, capabilities to simulate the wave propagation that occurs from UT can be a great benefit to the NDE process. Analytical approaches are often unable to provide an efficient prediction of wave propagation in realistic structures with complex geometries. Therefore, a predictive simulation is fundamental for effectively applying NDE and SHM. However, the balance of accuracy and efficiency in simulating Ultrasonic wave propagation in realistic structures is challenging. The finite element method (FEM) and the boundary element method (BEM) have often been preferred in previous studies to simulate the wave propagation in structures. For example, guidelines for modeling guided lamb wave propagation has been provided in previous studies $[24,3]$. In those studies FEM models were built using the commercial software ABAQUS/Explicit to simulate lamb wave propagation in thin plate structures. The central difference method was used for explicit time integration to provide a reasonable trade-off between computational expense and accuracy. Although explicit integration has the benefit of relatively low computational cost, instability of the numerical solution might occur. To try to ensure stability, a limit on the time increment is given as [3]:

$$
\triangle t \leq \frac{1}{20 f_{\max }}
$$

where $f_{\max }$ is the maximum frequency of the structure. To simulate the wave propagation accurately, the element size of the model is also restricted by the wavelength. Moser et al. recommend a minimum of 20 nodes per wavelength to ensure sufficient spatial resolution as $[25]:$

$$
L_{e} \leq \frac{\lambda_{\min }}{20}
$$

where $L_{e}$ is the element size and $\lambda_{\min }$ is the minimum wavelength of the structure.

The simulation of the excitation and receiver is an important part of simulating wave propagation-based NDT. Gresil et al. introduced a way to simulate the excitation of square and round piezoelectric wafer active sensors (PWAS) for actuation as 12 and 8 self-equilibrating 


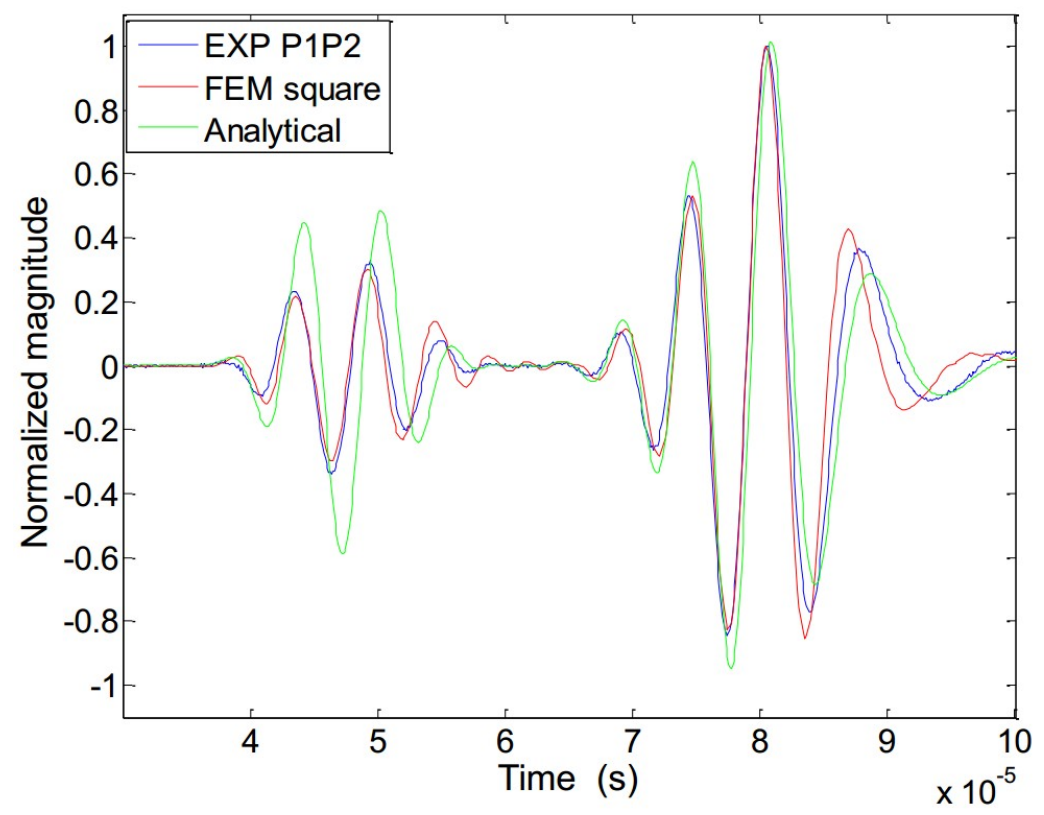

Figure 1.5: Comparison of a tone burst signal received from a finite element model using approximate excitation, as well as analytical and experimental results [3].

forces [3]. In that work a modulated sinusoidal tone burst of some limited number of cycles was used to simulate the pulse signal. This approximation of the excitation may cause a relatively small time shift between experimental, analytical, and numerical results. For example, Figure 1.5 shows the comparison of the signal received generated by the FEM using the approximate excitation, an analytical approximation, and experimental results. Additionally, there are several methods to simulate the PWAS receiver. One approximation could be recording the in-plane strain at the center of the PWAS [24], since the output signal of the piezoelectric sensor is directly related to the mechanical strain.

\subsubsection{Inverse Problems in Structural Dynamics}

1.3.4.1 Inverse Problem Overview: It is always significantly more challenging to solve an inverse problem than the corresponding forward problem. For instance, inverse problems in mathematics are uncountable and often have multiple solutions [26]. In structural dy- 


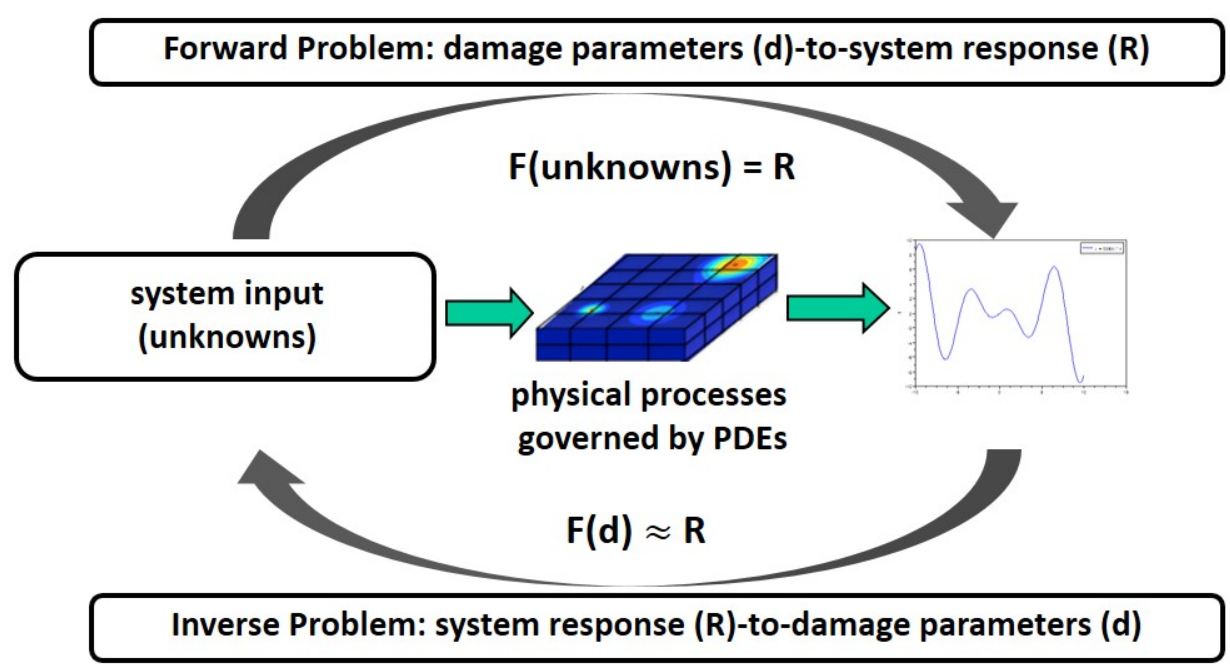

Figure 1.6: Schematic of inverse problems in structural dynamics.

namics, forward problems typically consist of calculating the response, such as displacement, strain, velocity, etc., of a given structural system knowing the material properties, geometry, and boundary conditions. In contrast, inverse problems include deducing unknown material properties, geometry, and/or boundary conditions of a structural system from some set of responses. Another definition of inverse problems for damage characterization was introduced in [12]. Inverse problems typically estimate the unknown material properties (such as material stiffness distribution) from the system response, while a forward problem measures the response of the systems with unknown material properties. The NDT techniques described in the previous sections, such as UT, TIR and AE, are used to obtain the system responses as would relate to the forward problems and used as input for the inverse problems. Figure 1.6 illustrates the concept of inverse problems in structural dynamics.

A large amount of focus has been placed on different inverse solution methods for material characterization or damage identification in various fields $[4,5,6,7,8]$. One of the common computational approaches to solve these inverse problems is based on updating structural model parameters (e.g., stiffness, mass, and damping) within a representation of the structural system until the dynamic response experimentally measured for that system is sufficiently approximated. As described in the previous section, traditional FEM and BEM 
are commonly applied to simulate NDT responses for a variety of systems. The reliability of these computational inverse solution approaches relies on the accuracy and efficiency of the computational model representing the physical behavior of the system subject to the NDT. If an accurate computational representation can be created, an optimization problem can then be arranged to minimize the difference between the reproduced dynamic responses from the computational representation and the response experimentally measured from the NDT.

The modeling assumptions and the parameterization of the unknown fields to be determined play an important role in this solution process, and there have been a wide variety of applications that utilize an equally wide variety of modeling approaches and parameterizations. For example, for inverse characterization of damage or degradation, the damage or "unhealthy" features of a structure could be predefined to manifest as changes in the material properties, such as Young's modulus, mass, and/or damping. In [4] Gaussian radial basis functions (GRBF) were used to represent the spatial variation of Young's modulus in unhealthy tissue. As such, the spatial distribution of Young's modulus was expressed as:

$$
E(\vec{x})=E_{o}+\sum_{i=1}^{n} \lambda_{i} \exp \left(-\frac{\left\|\vec{x}-\vec{\xi}_{i}\right\|^{2}}{c_{i}^{2}}\right),
$$

where $E_{o}$ is the healthy Young's modulus, $\vec{\xi}_{i}$ are the coordinates of the GRBF centers, $c_{i}^{2}$ is the parameter that control the size of the GRBFs, $\lambda_{i}$ are the real-valued amplitude coefficients, and $\|\cdot\|$ is the standard $l_{2}$ norm. Notghi et al. also used a GRBF representation of Young's modulus distribution for inverse characterization of damage in plate structures $[27]$.

The objective function that quantifies the error in the computational representation of the structural system based on different geometric and physical properties of also significantly affects the accuracy and efficiency of the inverse solution procedure. Most often these objective functions are simply defined as some suitable metric norm of the error between the simulated and the experimental NDT responses. In [4] the inverse problem was cast as an optimization problem to characterize the Young's modulus distribution from AE frequency 
responses, the FEM was used to simulate the system's responses, and the objective function quantifying the error between the simulated and experimental responses was defined as:

$$
J=\sqrt{\sum_{j=1}^{l} \sum_{k=1}^{m}\left(r_{j k}^{E x p}\left(\overrightarrow{x_{j}}, \omega_{k}\right)-r_{j k}^{F E A}\left(\overrightarrow{x_{j}}, \omega_{k},\{c\},\{\hat{E}\},\{\zeta\},\left\{E_{0}\right\}\right)\right)^{2}}
$$

where $r^{E x p}$ and $r^{F E A}$ are the experimental and finite element approximated responses, respectively, $\omega_{k}$ is the excitation frequency, $\{c\}$ ad $\{\zeta\}$ are the parameters representing the size and location of the degradation, respectively, and $\{\hat{E}\}$ is the Young's modulus at the degradation center. A similar relative error norm between experimental and approximate frequency response was defined and utilized for inverse damage characterization in [27]. Alternatively, a more physical equation (e.g., based on a boundary value problem) is another option to create as objective function. For instance, in [6] an inverse transient heat conduction problem was used to identify the unknown thermal properties of a system by minimizing the following function based on conservation of energy:

$$
J(\rho c, \lambda)=\frac{1}{2} \int_{0}^{t_{f}}\left\|\rho c C \frac{d \theta}{d t}+\lambda K \theta(t)-F\right\|^{2} d t .
$$

1.3.4.2 Optimization Approaches: Provided with a suitable objective function, optimization techniques can be used to minimize the objective function and identify an estimate to the inverse solution. Generally, there are two main categories of optimization techniques, gradient-based methods [28, 29] and non-gradient-based methods [30, 31, 32], with both having certain advantages and shortcomings. Generally, gradient-based methods to minimize a function can be described as follows:

1. Estimate a starting point (i.e., an initial estimate of the unknown properties), $x_{0}$, and set $k=0$.

2. Test for convergence - Compute the value of the objective function for $x_{k}$. If the criteria for convergence is satisfied (i.e., the error is low enough), the algorithm can STOP and give $x_{k}$ as the solution. Otherwise, continue to the next step.

3. Determine the next search point - Compute the gradient of the objective function at current point, $x_{k}$. Use the gradient to calculate a step size and direction and use this step information to update the solution estimate. 
4. Update the variables - Set $x_{k+1}$ to be the current solution estimate and set $k=k+1$, and RETURN to Step 2.

The gradient-based family of methods includes the steepest descent method, the conjugate gradient method, Newton's method, modified Newton's method and trust region methods, among others. Gradient-based methods typically require a much smaller number of iterations (and therefore computational time) to converge comparing to the non-gradient-based methods. However, global search capabilities for gradient-based methods is limited and they are susceptible to premature convergence to local minima. The initial estimate of the solution will therefore significantly affect the ability of the algorithm to find an accurate solution. Furthermore, there is added complexity in gradient-based methods due to the need to compute the gradients of the objective function, potentially including first or higher derivatives of the objective function.

In contrast, non-gradient-based methods typically have a better ability to find global minima, but require a substantially larger number of iterations than gradient-based methods. In addition, the gradient of the objective function is obviously not required for non-gradientbased methods, and typically only evaluation of the objective function itself is necessary. Grid search methods, stochastic and nonlinear simplex, and genetic algorithms (GA) are all examples of non-gradient-based approaches. As a non-gradient-based algorithm that has been employed in several inverse problem solution procedures, the theory of GA will be briefly presented in the following.

Figure 1.7 illustrates a flowchart for a conventional GA. The fundamental concept of GA comes from the processes observed in natural evolution. GA, which is classified as evolutionary algorithms, imitates the evolutionary process of a population, and includes the core operators of selection, crossover, and mutation to provide solution estimates. These three basic operators in GA can be described as follows:

Slection : Selection is the process of survival of the fittest. In other words, the individuals that have a better fitness value (i.e., lower objective function value) will have a higher possibility to be selected to survive and/or generate offspring. There are several different 


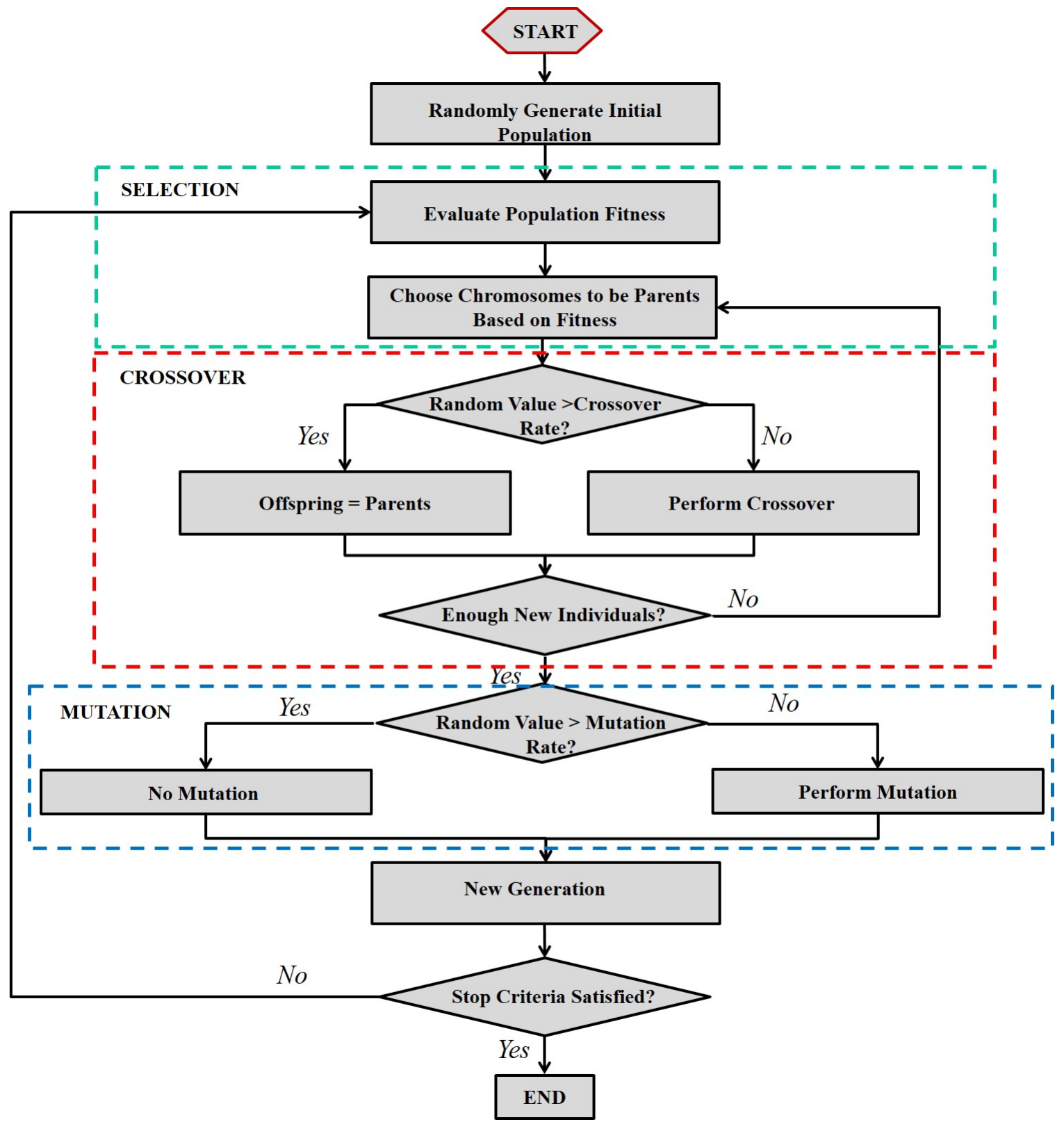

Figure 1.7: Flowchart of a conventional Genetic Algorithm (GA). 
ways to achieve selection, such as rank selection, tournament selection, elite selection, and roulette selection. Further discussion of selection techniques are presented in [31].

Crossover : Crossover is the process to combine genetic information from parents to produce offspring. The objective of this process is to reproduce an "improved" combination of genetic information. A simple way to achieve this is to copy genetic information from one part of a parent's information and copy the rest from the other parent. One important point is that no new genetic information is created in crossover.

Mutation : Similar to natural mutation, this process randomly modifies some part of the genetic information of an individual. Mutation decreases the possibility for an entire population converging to a local solution, as mutation guarantees some amount of genetic diversity for the next generation. Note that the mutation rate is usually much smaller than the crossover rate.

In order to combine the benefits and relieve the shortcomings of gradient-based methods and non-gradient-based methods, sometimes hybrid approaches containing both gradientbased and non-gradient-based algorithms are used. Examples for damage characterization problems that utilize various optimization approaches in a wide variety of structures are presented in previous studies $[4,33,11,10]$. Although a large amount of work has shown the ability of these optimization approaches to solve inverse problems in characterization of structures, there are some common challenges that still exist, particularly the potential computational expense in practice. The FEM applied to analyze NDT in realistic complex structures often requires substantial computational time and power. Applying limiting assumptions, such as that only one or a small number of material properties are changed by the damage, can simplify the optimization process, but in turn can decrease the accuracy of the inverse solution. Alternatively, designing a NDT that improves the solvability of the inverse problems can also substantially decrease the cost of the associated optimization process. 


\subsection{OPTIMAL NONDESTRUCTIVE TEST DESIGN}

Optimal NDT design, including selecting controllable features such as actuator location, sensor orientation and location, and frequency, has the potential to go a long way in overcoming or at least relieving some of the challenges discussed in inverse damage and/or material characterization using computational inverse mechanics techniques. Furthermore, optimal NDT design can provide guidance for implementation of specific NDE, helping to answer questions such as how many sensors are needed to inversely characterize the material properties for a particular NDT system. In recent years, several optimal NDT design approaches for damage detection and identification were introduced and developed [34, 35, 36, 37, 27, 38, 39, 40]. However, the vast majority of the existing NDT design approaches focus on optimizing the number and location of sensors alone.

One essential part of all current methods for NDT design is the definition of an appropriate metric to evaluate the quality of the NDT parameters (e.g., sensor locations), and a wide variety of metrics have been used. In [38, 39] a methodology to optimize damage detection and localization applications was introduced based on minimizing the information entropy with respect to the uncertainty in the model parameters. GA was employed to search for the "best" locations of sensors with the minimum information entropy. The approach for optimal sensor allocation proposed in [34] used the probability of damage identification as the metric to evaluate the sensor allocation. A probability distribution function for the optimal sensor allocation was computed based on the weights in a neural network that was trained to represent the behavior of the SHM system. The determinant of the target mode information matrix is another option that has be used to measure the quality of a sensor array [36]. In the process to find the optimal sensors locations, this method iteratively expands the small initial set of sensors instead of traditionally discarding candidates from a larger set. For the most part, these previous studies only considered (and many are only applicable to) the number of the sensors and their distribution as the variables in the NDT optimization. However, the aspects such as actuator location, direction, frequency, and test period of a NDT also have significant effect on the quality of the measurement information. Moreover, the examples presented in most of the current work provided limited support for 
the applicability to general cases. In order words, the techniques were only applicable to the specific structural systems and property that was discussed.

Raich and Liszkai [40] proposed a multi-objective optimization approach for NDT design that maximizes the sensitivity of the frequency response collected by sensors to the changes in the material properties, while also minimizing the number of required sensors. Both layouts of sensors and actuators were optimized in the examples presented. More specifically, case studies of structural damage characterization in beam and frame structures were presented to support this method for optimization of frequency response function-based NDT. The concept of maximizing sensitivity was extended to be applicable to a larger variety of NDT systems in [27], including solid continua in particular. Furthermore, [27] added in an objective to minimize the information redundancy collected by all sensors along with maximizing the sensitivity of the NDT design. Inverse characterization of the Young's modulus distribution of beam and thin plate structures was considered with simulated NDT and NDE problems, and the results further supported the concept of maximizing sensitivity for optimal NDT design. However, both studies applied this concept only in the frequency domain for frequency response function-based NDT.

In order to further explore the potential of this sensitivity maximization concept for optimal NDT design, the present work introduces a general approach to optimal wave propagation-based NDT design for damage characterization applications. More specifically, the objective of this work is to improve the accuracy and efficiency of the damage characterization process by optimizing the parameters of the NDT, such as the locations of sensors and actuators. The NDT design approach developed is based on maximizing the sensitivity of the NDT response measurements to changes in the material properties to be determined by the evaluation, while simultaneously minimizing the redundancy of response measurements. In particular, the concept of maximizing sensitivity with respect to the damage parameters $[27,40]$ is extended to wave propagation-based NDT. The forward problem describing the specific wave propagation-based NDT considered in presented next, followed by the inverse characterization problem formulation, and then the presentation of the NDT optimization techniques. Lastly, two simulated case studies are presented to evaluate the performance of 
the optimal wave propagation-based NDT design approach, which is followed by the concluding remarks. 


\subsection{FORWARD PROBLEM SIMULATION USING THE FINITE ELEMENT METHOD}

\subsection{WAVE PROPAGATION IN ELASTIC SOLIDS}

When a deformation wave propagates in a structure oscillations occur in space as well as in time. While this behavior can be analyzed in either the frequency domain or the time domain depending on the objectives of the analysis, only transient wave propagation was considered in this work. Naturally, transient vibration in a structure is generated by some transient excitation signal, and the nature of this vibration will significantly depend on the nature of this input signal. Some excitation examples include a transient signal of 5 cycles of a sine wave at $50 \mathrm{kHz}$ or a 5 cycle tone burst signal with a center frequency of $50 \mathrm{kHz}$, which are shown in Figure 2.1a and 2.1b, respectively.

Dispersion was not considered while simulating wave propagation in this work. Therefore, the transient signal in Figure 2.1a would travel through a structure at constant speed with the same waveform if the structure were homogeneous and isotropic. Alternatively, the tone burst signal shown in Figure 2.1b, which contain a narrow band of frequencies, would propagate through a structure as a group of waves with a group speed. Although the wave propagation behavior due to a tone burst signal is more complex, the group speed would be constant as dispersive waves are not considered here. In wave propagation-based UT, tone burst generators are commonly employed to excite structures, and these tone burst signals are well accepted in UT applications since they combine the features and benefits of pulse along with continuous tones. 


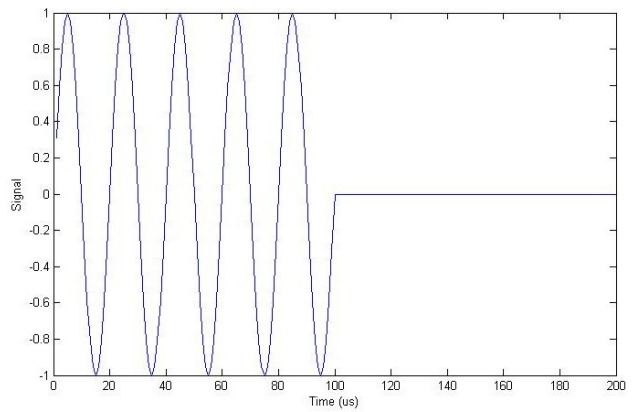

(a)

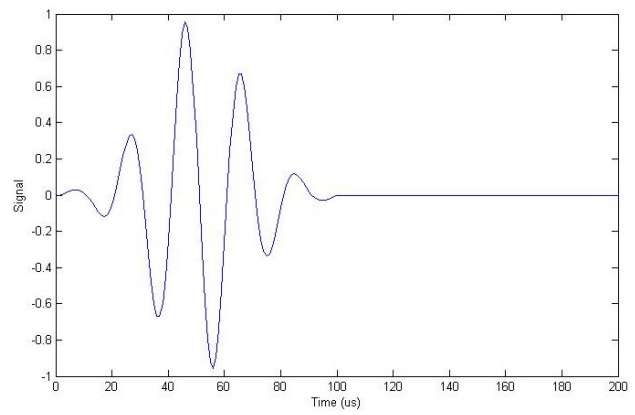

(b)

Figure 2.1: Time domain transient signal for (a) 5 cycles of a $50 \mathrm{kHz}$ sine wave and (b) 5 cycles of a tone burst with $50 \mathrm{kHz}$ center frequency.

\subsection{FINITE ELEMENT FORMULATION FOR SOLID DYNAMICS}

The dynamic systems discussed herein can be described by boundary-value problems (BVP), which consist of a system of partial differential equations (PDEs) derived from the conservation of linear momentum combined with appropriate boundary conditions. In this work, the body forces that arise from gravity were not considered. Therefore, the governing equilibrium equations for the BVP can be given as:

$$
\begin{aligned}
& \rho \ddot{u}_{x}-\left(\frac{\partial \sigma_{x x}}{\partial x}+\frac{\partial \sigma_{z x}}{\partial z}+\frac{\partial \sigma_{x y}}{\partial y}\right)=0, \\
& \rho \ddot{u}_{y}-\left(\frac{\partial \sigma_{y y}}{\partial y}+\frac{\partial \sigma_{z y}}{\partial z}+\frac{\partial \sigma_{x y}}{\partial x}\right)=0, \\
& \rho \ddot{u}_{z}-\left(\frac{\partial \sigma_{z z}}{\partial z}+\frac{\partial \sigma_{z y}}{\partial y}+\frac{\partial \sigma_{x z}}{\partial x}\right)=0,
\end{aligned}
$$

or

$$
\rho \overrightarrow{\ddot{u}}(\vec{x}, t)-\nabla \cdot \sigma(\vec{x}, t)=\overrightarrow{0} \text { in } \Omega \times I,
$$

where $\rho$ is the solid mass density, which was assumed to be constant here, $\vec{x}$ denotes the position vector, $\mathrm{t}$ is the time, $\overrightarrow{\ddot{u}}(\vec{x}, t)$ is the acceleration vector, $\sigma(\vec{x}, t)$ is the stress tensor, $\Omega$ is the given solid domain, and $I$ is the time domain. Since the displacements and strains 
generated by an ultrasonic excitation in UT are relatively small, the small strain and deformation assumptions can be applied. Therefore, the strain-displacement relationship can defined as:

$$
\epsilon(\vec{x}, t)=\frac{1}{2}\left\{\nabla \vec{u}(\vec{x}, t)+[\nabla \vec{u}(\vec{x}, t)]^{T}\right\}
$$

where $\epsilon(\vec{x}, t)$ is the strain tensor. Then, assuming that the structures considered would behave linearly elasticity, the stress-strain relationship can be written as:

$$
\sigma(\vec{x}, t)=C^{I V}: \epsilon(\vec{x}, t)
$$

where $C^{I V}$ is the $4^{t h}$-order elasticity tensor.

Based on Cauchy's stress principle, the natural (i.e., traction) boundary conditions can be defined as:

$$
\sigma(\vec{x}, t) \cdot \vec{n}(\vec{x})=\vec{\tau}(\vec{x}, t) \text { on } \Gamma_{N} \times I
$$

where $\vec{n}$ is the unit outward normal vector to the surface, $\vec{\tau}(\vec{x}, t)$ denotes the traction vector, and $\Gamma_{N}$ is the portion of the boundary of the solid with traction specified. In addition, the essential (i.e., displacement) boundary conditions are defined as:

$$
\vec{u}(\vec{x}, t)=\vec{g}(\vec{x}, t) \text { on } \Gamma_{D} \times I,
$$

where $\vec{u}(\vec{x}, t)$ is the displacement vector and $\Gamma_{D}$ is the portion of the boundary of the solid with displacement specified.

Two initial conditions, typically relating to displacement and velocity, are required to solve the BVP in the time domain (i.e., the initial boundary value problem) and can be given as:

$$
\vec{u}\left(\vec{x}, t_{0}\right)=\vec{u}_{0}(\vec{x}) \text { in } \Omega
$$

and

$$
\overrightarrow{\dot{u}}\left(\vec{x}, t_{0}\right)=\overrightarrow{\dot{u}}_{0}(\vec{x}) \text { in } \Omega \text {. }
$$


The initial displacement and velocity were consistently set to be zero for the present study, which is often the case.

For the present work the PDEs above were simplified to a two-dimensional (2-D) form based on the plane stress assumption for the solids considered (note that all case studies considered herein were thin plates for which plane stress would apply). As such, the stress components of the structures in the third direction, $\sigma_{z z}, \sigma_{z x}$, and $\sigma_{z x}$, were all assumed to be zero, and the stress-strain relationship could be reduced to 2-D. Thus, the stress-strain relationship for the nonzero components could be written as:

$$
\left[\begin{array}{c}
\sigma_{x x} \\
\sigma_{y y} \\
\sigma_{x y}
\end{array}\right]=\left[\begin{array}{ccc}
2 \mu+\lambda & \lambda & 0 \\
\lambda & 2 \mu+\lambda & 0 \\
0 & 0 & \mu
\end{array}\right]\left[\begin{array}{c}
\epsilon_{x x} \\
\epsilon_{y y} \\
2 \epsilon_{x y}
\end{array}\right],
$$

where $\lambda$ is lamé's first parameter and $\mu$ is lamé's second parameter (also referred to shear modulus G). These two parameters can also be expressed in terms of the Young's modulus $(E)$ and Poisson's ratio $(\nu)$ for plane stress as:

$$
\begin{gathered}
\lambda=\frac{E \nu}{1-\nu^{2}} \\
\mu=\frac{E}{2(1+\nu)}
\end{gathered}
$$

The FEM formulation was developed based on the weak-form Galerkin approach. For this approach a virtual displacement (i.e., weight function or trial function) vector $\vec{\omega}(\vec{x}, t)$ is defined that satisfies homogeneous essential boundary conditions anywhere Dirichlet conditions are specified in the BVP. The dot product can then be taken between the governing equation (Eqn. (2.2)) and this virtual displacement vector, and through some manipulation, the weak form of the governing BVP can be derived as:

$$
\begin{array}{r}
\int_{\Omega} \rho \vec{\omega}(\vec{x}, t) \cdot \vec{u}(\vec{x}, t) d \Omega+\int_{\Omega} \delta \vec{\epsilon}(\vec{x}, t) \cdot \vec{\sigma}(\vec{x}, t) d \Omega \\
-\int_{\Gamma_{N}} \vec{\omega}(\vec{x}, t) \cdot \vec{\tau}(\vec{x}, t) d \Gamma_{N}=0
\end{array}
$$

where $\delta \vec{\epsilon}(\vec{x}, t)$ is the virtual strain vector that is derived from the virtual displacement vector. 
The Galerkin FEM approach approximates the displacement and virtual displacement fields with identical basis functions as:

$$
\vec{u}(\vec{x}, t)=[N(\vec{x})]\left\{u^{e}(t)\right\}
$$

and

$$
\vec{u}(\vec{x}, t)=[N(\vec{x})]\left\{\omega^{e}(t)\right\}
$$

where $\left\{u^{e}(t)\right\}$ and $\left\{\omega^{e}\right\}$ are the nodal displacement and nodal virtual displacement vectors (i.e., coefficient vectors), respectively, and $[N(\vec{x})]$ is the shape function matrix (i.e., matrix of basis functions). Based on the above expressions, the strain-displacement relationship can be written as:

$$
\vec{\epsilon}(\vec{x}, t)=[B(\vec{x})]\left\{u^{e}(t)\right\}
$$

and

$$
\delta \vec{\epsilon}(\vec{x}, t)=[B(\vec{x})]\left\{\omega^{e}(t)\right\}
$$

where $[B(\vec{x})]$ is the matrix of spatial derivatives of the shape functions.

Eqns. (2.13)-(2.16) can be substituted into Eqn. (2.12) and the arbitrary virtual displacement vector can be eliminated to produce a global linear system of equations as (neglecting the details of the concepts of elements and assembly here for brevity, and just showing the quantities as summations over all elements):

$$
[M]\{\ddot{u}(t)\}+[K]\{u(t)\}=\{F(t)\}
$$

where

$$
\begin{aligned}
& {[M]=\sum_{\text {elements }} \int_{\Omega^{e}} \rho[N(\vec{x})]^{T}[N(\vec{x})] d \Omega^{e},} \\
& {[K]=\sum_{\text {elements }} \int_{\Omega^{e}}[B(\vec{x})]^{T}[C][B(\vec{x})] d \Omega^{e},}
\end{aligned}
$$


and

$$
\{F(t)\}=\sum_{\text {elements }} \int_{\Gamma_{N}^{e}}[N(\vec{x})]^{T} \vec{\tau}(\vec{x}, t) d \Gamma_{N}^{e} .
$$

In the above global system of equations, $[M]$ is the global mass matrix, $\{F(t)\}$ is the external force vector, $[K]$ is the global stiffness matrix and $[C]$ is the elasticity matrix (as shown in Eqn. (2.9)).

\subsection{NUMERICAL TIME INTEGRATION}

\subsubsection{Explicit Time Integration}

To solve the ordinary differential equations with respect to time developed in previous section (Eqn. (2.17)), a suitable time integration approach is needed. In general, there are two groups of direct time integration methods that are used to solve transient wave propagation and structural dynamics problems: explicit and implicit methods [41]. A time integration method is explicit if the inverse of an effective stiffness matrix is not required, otherwise, the time integration method is implicit. Both explicit and implicit time integration methods have their own benefits and shortcomings. Since implicit methods need to calculate an inverse of the effective stiffness matrix, they require much more computational time and power compared to explicit methods. However, the size of the integration time step in implicit methods is unrestricted in terms of stability, and only depends on the physical problem and desired time resolution for accuracy. Alternatively, explicit methods commonly avoid calculation of any matrix inverse by using a diagonalized mass matrix (i.e., lumped mass matrix) instead of a consistent mass matrix (as shown in Eqn. (2.18)), and are therefore highly computationally efficient. Unfortunately, the solution stability is conditional when using explicit methods. Therefore, a limitation on the size of the time incrementation based on the physics of the system is typically enforced to ensure a stable solution. Yet, wave propagation analysis based on UT naturally requires a relatively small time step (due to the

high frequency of excitation) to accurately resolve the behavior, regardless of the integration 
method. Therefore, it is logical to apply explicit direct time integration for work such as the current study, for the simulation of high-frequency ultrasonic wave propagation in structures.

The explicit central difference direct time integration method was used to solve the governing finite element equation described in Eqn. (2.17) for the present work, and is briefly described in the following. Firstly, applying the central difference method the displacement, velocity, and accelerations at various times can be related to one another as:

$$
\dot{u}^{\left(t+\frac{\Delta t}{2}\right)}=\dot{u}^{\left(t-\frac{\Delta t}{2}\right)}+\Delta t \ddot{u}^{(t)}
$$

and

$$
u^{(t+\Delta t)}=u^{(t)}+\Delta t \dot{u}^{\left(t+\frac{\Delta t}{2}\right)}
$$

where $t$ denotes the current analysis time, $\Delta t$ is the time step size, and the superscript references the value of time for a specific field. The governing finite element equations are then evaluated at time $t$ as:

$$
[M]\left\{\ddot{u}^{t}\right\}+[K]\left\{u^{t}\right\}=\left\{F^{t}\right\},
$$

where $[M]$ is now the lumped mass matrix. Note that there are several methods to lump the mass matrix into a diagonal matrix, such as physical lumping and row summation methods. For simplicity, the diagonal mass matrix herein was lumped by a row summation method, such that $M_{i i}^{L}=\sum_{j=1}^{n} M_{i j}^{C}$, where $\left[M^{L}\right]$ is lumped mass matrix and $\left[M^{C}\right]$ is the consistent mass matrix. Because the displacement at time $t$ is known (given by the initial conditions at the first step), the acceleration at time $t$ can be calculated from Eqn. (2.23), and then the displacement and velocity can be updated based on Eqns. (2.21) and (2.22). For the first time step of the analysis the velocity at time $-\frac{\Delta t}{2}$ is unknown, but can be avoided by updating the velocity for the first step by:

$$
\dot{u}^{\left(\frac{\Delta t}{2}\right)}=\dot{u}^{(0)}+\frac{\Delta t}{2} \ddot{u}^{(0)}
$$




\subsubsection{Stable Time Increment}

The conditional stability and stable time increment limitation for explicit time integration methods were firstly proposed by [42]. To help prevent unstable numerical results, a critical time increment can be defined as (details for the derivation of the critical time increment can be found in [43]):

$$
\Delta t \leq \frac{2}{\omega}
$$

where $\omega$ is the natural frequency calculated from the following eigenvalue equation:

$$
[K] \vec{x}=\omega^{2}[M] \vec{x}
$$

In the above equation, $[K]$ and $[M]$ again denote the global stiffness matrix and mass matrix, respectively. However, this eigenvalue problem for complex systems can be difficult to solve. Thus, the critical time increment can be estimated instead by calculating the maximum eigenvalue of a single element as:

$$
\Delta t \leq \frac{2}{\omega_{\max }^{e}},
$$

where $\omega_{\max }^{e}$ is the maximum element eigenvalue. In addition, as was discussed in the previous Chapter, the highest frequency of the ultrasonic wave also limits the time incrementation to ensure accurate resolution of the system responses. Therefore, the time increment for the simulation of the ultrasonic wave propagation herein was limited by the minimum of the two requirements (the maximum element eigenvalue and the highest frequency of the ultrasonic wave). Thus, the smaller time increment calculated between Eqn. (1.4) and Eqn. (2.27) was set to be the critical time increment. 


\subsection{VERIFICATION OF THE FORWARD MODEL}

Before any extensive forward or inverse analyses are performed, it is critical to verify the accuracy of the simulation tools to be used to estimate the dynamic response of solids. For the present work the open source finite element library FEniCS [44] was employed to build a finite element analysis (FEA) software to solve the transient BVP presented above for all case studies considered. In order to verify the solution (i.e., check that the mathematics are accurately solved), a two dimension finite element model was built and analyzed using the FEniCS software developed and compared to the same analysis performed with the well-developed and trusted commercial finite element software ABAQUS [45].

A $1 m \times 1 m \times 0.02 m$ aluminum plate structure was considered here, shown schematically in Figure 2.2. The bottom boundary of the plate was set to be fixed and the remaining three sides were free to deform. A $1 k P a$ harmonic load with an excitation frequency of $5 k H z$ was applied uniformly to the top surface of the plate, and the plane stress condition was assumed. The plate was discretized by linear triangle elements with a height and width of $2 \mathrm{~mm}$. As discussed, explicit time integration was used with a fixed time increment that was set to satisfy Eqn. (1.4) and Eqn. (2.27).

A comparison of the vertical displacement responses for two representative spatial points over time from the results of the two finite element softwares (i.e., the one created in FEniCS and the ABAQUS software) is shown in Figure 2.3a and Figure 2.3b. It can be observed that the results of the two finite element softwares were nearly exactly the same. Furthermore, the relative difference for all nodal vertical displacements at every time step between ABAQUS and the FEniCS software was calculated as $1 \%$ based on:

$$
D_{\text {error }}=\frac{1}{N_{t} N_{e}} \sum_{i=1}^{N_{e}} \sum_{j=1}^{N_{t}}\left|\frac{r^{A B Q}\left(\vec{x}_{i}^{e}, t_{j},\right)-r^{F E n}\left(\vec{x}_{i}^{e}, t_{j}\right)}{r^{A B Q}\left(\vec{x}_{i}^{e}, t_{j}\right)}\right|,
$$

where $|\cdot|$ denotes the absolute value, $r^{A B Q}\left(\vec{x}_{i}^{e}, t_{j}\right)$ and $r^{F E n}\left(\vec{x}_{i}^{e}, t_{j}\right)$ denote the ABAQUS and FEniCS simulated responses, respectively, $N^{e}$ is the total number of elements, and $N^{t}$ is the total number of time steps. Based on these results, the FEniCS code can be said to be verified to some extent for the simulation of wave propagation. Since the implementation of varying parameterizations of material property distributions is considerably easier within 


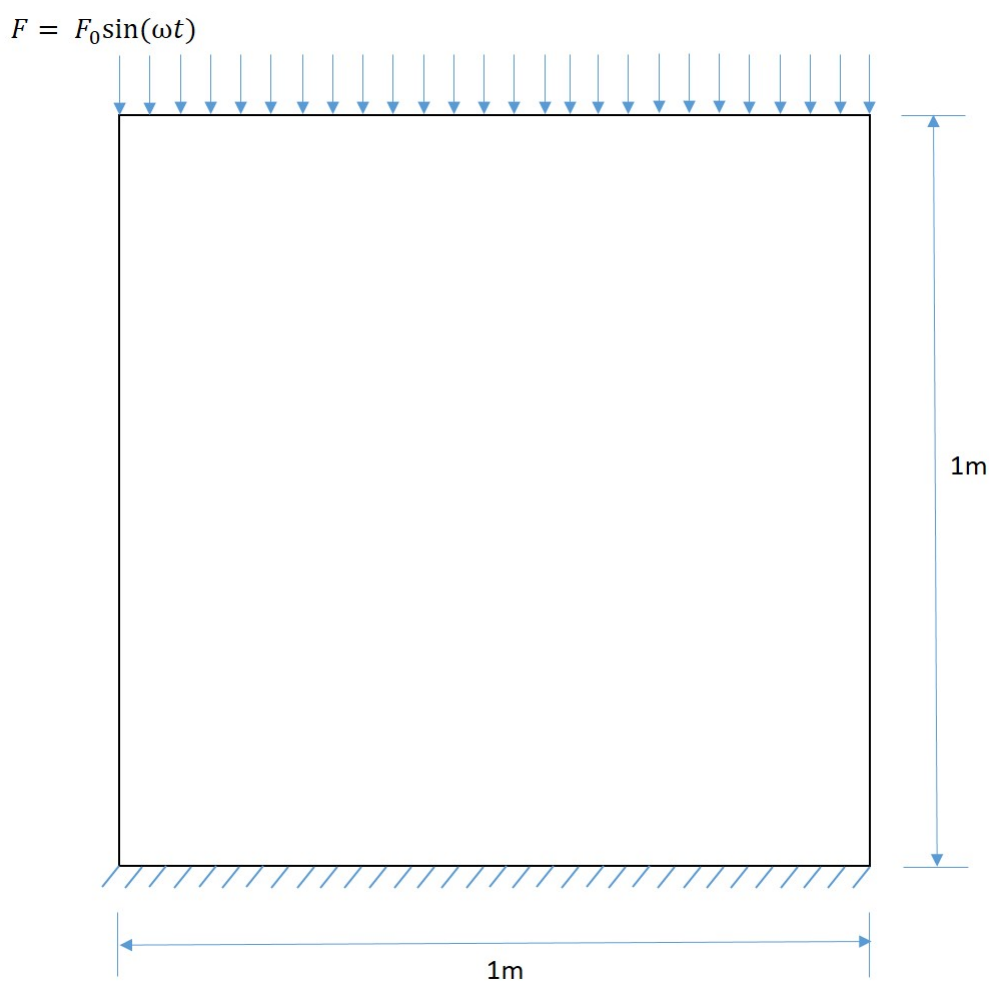

Figure 2.2: Schematic for the verification example. 


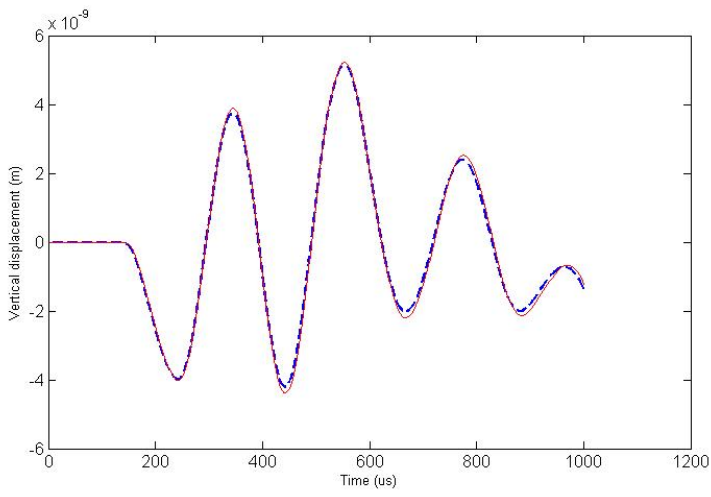

(a)

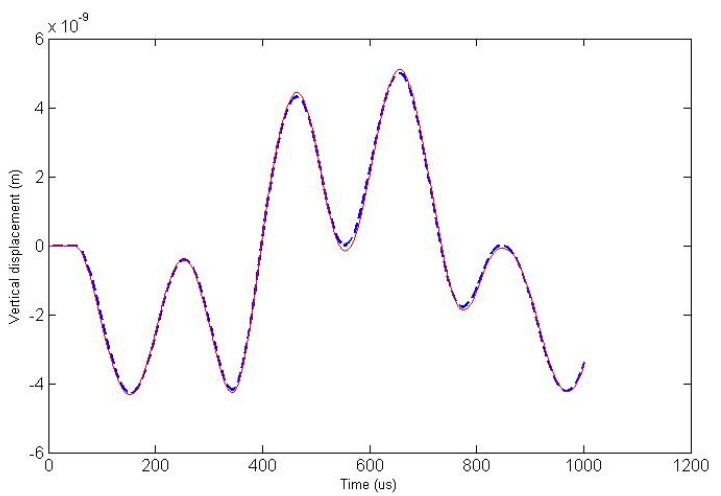

(b)

Figure 2.3: Comparison of the vertical displacement between ABAQUS (red solid line) and FEniCS (blue dash line) (a) at coordinate $(0.25 \mathrm{~m}, 0.25 \mathrm{~m})$ and (b) at coordinate $(0.75 \mathrm{~m}, 0.75 \mathrm{~m})$. 
the FEniCS environment than for ABAQUS (which is relatively important for a material characterization process), the FEniCS software developed was applied as the primary forward simulation tool for all of the following case studies. 


\subsection{INVERSE CHARACTERIZATION SOLUTION STRATEGY}

\subsection{INVERSE PROBLEM FORMULATION}

As described in the introduction, damage and/or material characterization of structural components using NDT approaches can be classified as an inverse problem. The inverse problem herein can be specifically stated as inversely characterizing the material properties relating to damage (including location and severity) in structural components using the timeresponse obtained from UT approaches. As such, once a reliable finite element model is built to accurately predict the structural behavior of UT (as described in the previous section), this inverse characterization problem can be cast as an optimization problem to minimize the difference between the experimental measurements from UT and responses predicted by the FEA. There are generally three steps to this optimization-based inverse solution approach:

1. Define the parameterization of the damage/material to be determined and identify any restrictions on these parameters, such as the physically admissible range.

2. Define an objective function that suitably quantifies the difference between the responses predicted by the numerical model for a given set of damage/material parameters and the experimentally measured UT data.

3. Employ an optimization algorithms to estimate the set(s) of material parameters that minimize the objective function.

The objective function that is defined based on some chosen geometric and physical prop-

erties of the structural system considered significantly affects the accuracy and efficiency of the inverse solution procedure. The basic example that will be considered in the present work is to simply apply some suitable metric norm to quantify the error between the sim- 
ulated and experimental UT measurements. Thus, assuming that the objective will be to determine the material stiffness distribution, an objective function for the inverse solution process can be written as:

$$
J(E(\vec{x}))=\left\|\frac{r^{E x p}(\vec{\psi})-r^{F E M}(\vec{\psi}, E(\vec{x}))}{r^{E x p}(\vec{\psi})}\right\|,
$$

where $r^{E x p}(\vec{\psi})$ and $r^{F E M}(\vec{\psi})$ are the time responses from the UT experiment and the finite

element model, respectively, $\vec{\psi}$ is the set of UT parameters (e.g., the excitation and sensor location, sensor orientation, center frequency of excitation signal, etc. ), and $\|\cdot\|$ is some appropriate metric norm that combine each measurement with respect to each time step into the total error function $J(E(\vec{x}))$. Potential physical restrictions of the parameter search domain will be discussed further according to specified case studies presented, and the following will outline details of a potential optimization approach for NDE.

\subsection{SURROGATE-MODEL ACCELERATED RANDOM SEARCH ALGORITHM}

In the present work, the surrogate-model accelerated random search (SMARS) optimization algorithm [13] was used to solve the NDE inverse problems by minimizing Eqn. (3.1). The SMARS algorithm combines the traditional random search $(\mathrm{RS})$ algorithm with a surrogatemodel (i.e., meta-model) method to accelerate the search while maintaining the ability to approximate a global solution. Many well developed stochastic search algorithms, such as GA and the RS algorithm have the potential for global search capabilities, but require a substantial number of iterations to reach a solution, which in this case means a large number of numerical analyses. Unfortunately, FEA of realistic structures can easily become complex and require a high computational expense. Therefore, hybrid algorithms, such as the SMARS algorithm are often more suitable for these types of inverse problems, since the local search component (the surrogate-model in this case) can significantly reduce the computational expense without sacrificing the solution capabilities significantly. The details 
of the RS approach and the surrogate-model method are introduced in the following to better illustrate the SMARS algorithm.

\subsubsection{Random Search Algorithm}

In general, the process of the RS algorithm can be simply described as follows [46, 30]: Firstly, search the solution domain by randomly generating a number of initial sets of parameter estimates and calculate the value of the objective function for each set; Secondly, based on the evaluation of the current set of solution estimates, randomly generate new sets of parameters near (in parameter space) to the current best parameter estimates; The new best set of parameter estimates is then found with respect to objective function, and the RS algorithm repeats this process of randomly generating parameter estimates until a stopping criterion is satisfied.

As a non-gradient-based optimization algorithm, RS algorithm has the potential to estimate a global solution. Moreover, the search process of the RS algorithm does not rely significantly on the size and quality of the initial estimates of parameters. However, the shortcoming of the RS algorithm is clearly that a large number of evaluations can be required to achieve convergence to a reasonable solution. Therefore, a pure RS algorithm may not be suitable when complex numerical models are used within the objective function to be minimized.

\subsubsection{Surrogate-Model Method}

The surrogate-model method is based on creating a low-order (i.e., computationally efficient) replacement (i.e., surrogate) model for the computationally expensive forward model (in this case finite element model) that can provide estimates of the response of this complex numerical model in fractions of a second [47,48]. There are a variety of approaches that have been used to build these replacement models, including machine learning tools, such as artificial neural networks and support vector regression. For a basic implementation, the surrogate-model method generates a set of inputs (i.e., parameter estimates for the inverse problem) and corresponding outputs (i.e., responses from the numerical model given the 
input parameter estimates). This input-output set is then used to "train" the surrogate model (applying some form of regression) to learn the relationship between the input and output and provide a high-speed prediction of the output given new input parameter values. After training, the surrogate-model is then employed to approximate the system responses in the optimization solution process (rather than the computationally expensive model). For example, the surrogate-model objective function, which now measures the difference between the experimental data and the surrogate-model approximation, can be defined as:

$$
J^{S M}(E(\vec{x}))=\left\|\frac{r^{E x p}(\vec{\psi})-r^{S M}(\vec{\psi}, E(\vec{x}))}{r^{\operatorname{Exp}}(\vec{\psi})}\right\|,
$$

where $r^{S M}(\vec{\psi}, E \vec{x})$ is the response computed by surrogate-model.

Since the computational cost of the surrogate model is relatively low, global search algorithms, such as GA, can now be suitable to minimize the surrogate-model objective function $J^{S M}(E(\vec{x}))$ and estimate a solution. However, although the surrogate-model method relieves the computational expense of the optimization problem, it is limited by the dataset used for training. Furthermore, since the dataset used to train the surrogate-model is bounded, the surrogate-model is only expected to be applicable for local optimization within the range of this dataset. For the present work, artificial neural networks (ANN) were used in the SMARS algorithm as the nonlinear mapping tools to build surrogate-models.

\subsubsection{SMARS algorithm}

Figure 3.1 shows a flowchart of SMARS algorithm that combines a RS algorithm with a surrogate-model method. The optimization process of the SMARS algorithm can be described by the following steps:

Step 1 Define the two stopping criteria for the algorithm, including the solution tolerance and the maximum number of iterations, and define the initial search boundaries for each parameter.

Step 2 Randomly generate a set of trial solutions in the initial solution domain and obtain the corresponding responses with the numerical model. 


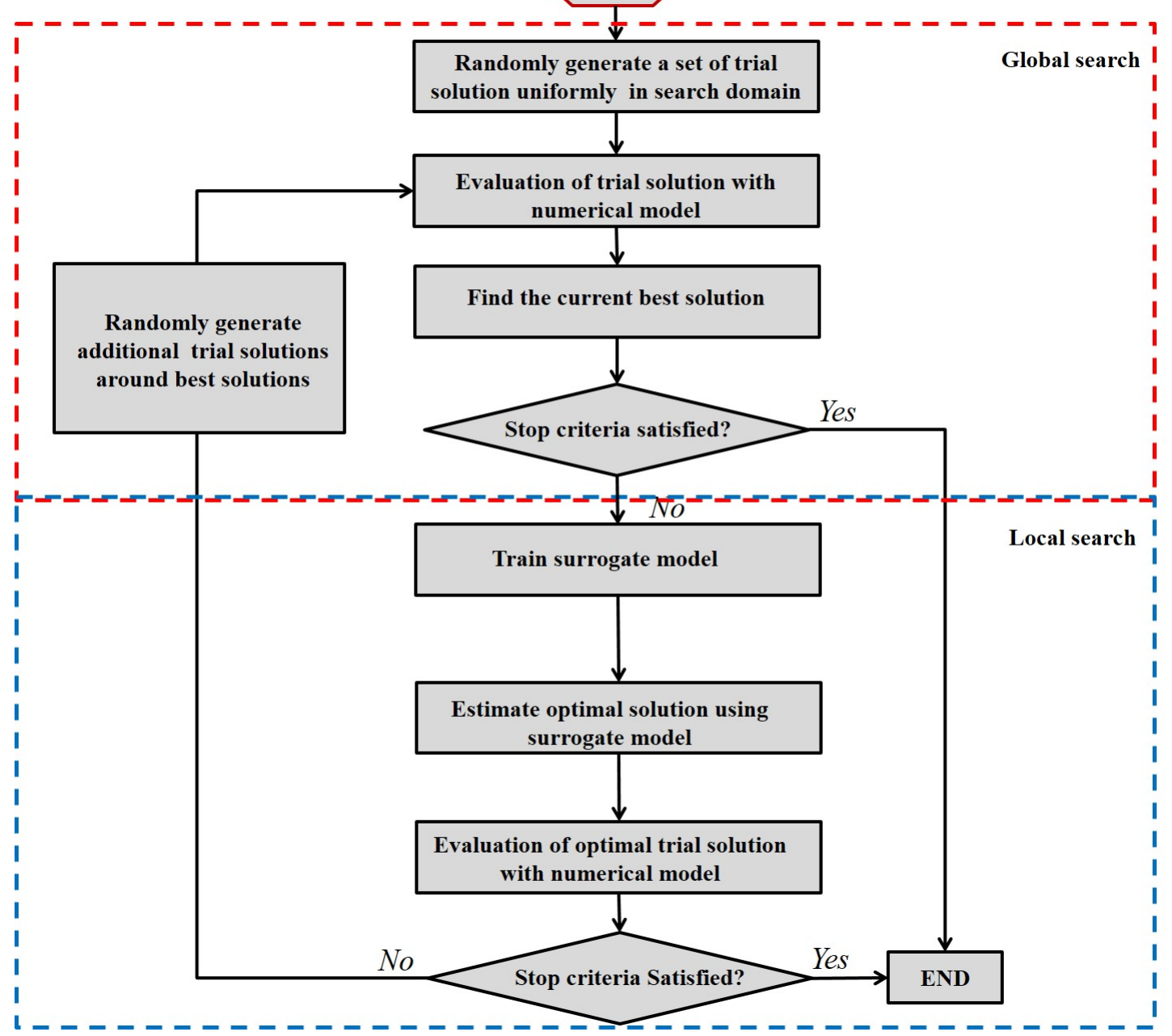

Figure 3.1: Flowchart of the surrogate-model accelerated random search algorithm, including the global search random search algorithm component and the local search surrogate-model method component. 
Step 3 Evaluate the objective function (Eqn. (3.1)) for the set of trial solutions and find the current best solution. If either stopping criteria is satisfied, STOP, otherwise, continue to the next step.

Step 4 Train the surrogate model with a subset of the current set of trial solutions.

Step 5 Find the approximate solution by minimizing the surrogate model error (Eqn. (3.2)).

Step 6 Compute the numerical response $\left(r^{F E M}(\vec{\psi}, E(\vec{x}))\right)$ of the approximate solution and the corresponding actual error $(J(E(\vec{x})))$. If the stopping criteria is satisfied, STOP, otherwise, continue to the next step.

Step 7 Add the approximate solution from the surrogate-model into the set of trial solution$\mathrm{s}$, and then randomly generate additional trial solutions around two selected solutions (referred to as "search poles") from the current set.

Step 8 Compute the numerical response $r^{F E M}(\vec{\psi}, E(\vec{x}))$ of the additional trial solutions. Find the best solution from the current set of trial solutions (including all randomly generated trial solutions and all surrogate-model approximate solutions) with respect to $J(E(\vec{x}))$. If either stopping criteria is satisfied, STOP, otherwise, GO TO Step 4.

In Step 5, the subset of trial solutions, which is used to train the ANN surrogate-model, is defined by specifying a distance around the current best solution in parameter space. In other words, the ANN is trained to map a smaller window around the current best solution instead of the entire parameters domain. In addition, a GA is applied to minimize the surrogate-model error $J^{S M}(E(\vec{x}))$ within this reduced search window in the present work. As stated previously, although a GA requires a large number of iterations to obtain an accurate global solution estimate, the computational cost of each iteration using the ANN surrogate-model is inexpensive, and the entire optimization process with the surrogate model has relatively low computational expense. Lastly, in Step 7, the two solutions around which new trial solutions are randomly generated are selected based on the following rules: (1) Select the best solution of the current set (i.e., the solution with the lowest corresponding $J(E(\vec{x})))$, and (2) Select the trial solution that is farthest from the first solution in parameter space, but among the best $20 \%$ of the current set of trial solutions in terms of $J(E(\vec{x}))$. 


\subsection{OPTIMIZATION OF NONDESTRUCTIVE TESTING}

Regardless of the type of NDT applied or the material properties and geometry of the structures considered, the quality of the measurement data used to estimate the unknown parameters significantly affects the accuracy and efficiency of the inverse characterization process. As discussed in the literature review, the concept of maximizing the sensitivity of the NDT responses collected with respect to changes in the material properties has been shown to be applicable to improve the capability of inverse characterization of unknown material properties for frequency response function-based NDT. The examples discussed included [40], which employed this concept as an objective along with another objective to minimize the number of the measurements to optimally determine the location of sensors and actuators. In addition, [27] presented a similar approach that also maximized the sensitivity of the response, while also minimizing the redundancy of the testing information to optimally design NDT. Alternatively, to further explore the potential of this maximum sensitivity concept, the present work introduces a generalized approach to optimal NDT design in the time domain for damage and/or material characterization with wave propagation-based NDT.

Similarly to the previous studies, the maximization of the response sensitivity of transient NDT responses to changes in material properties (e.g., Young's modulus) for NDT design can be based on an objective function of the form:

$$
C(\vec{\psi})=\left\|\frac{\partial r^{S M}(\vec{\psi}, E(\vec{x}), t)}{\partial E(\vec{x})}\right\|_{\Omega \times I},
$$

where $r^{S M}(\vec{\psi}, E(\vec{x}), t)$ is the simulated transient NDT responses, $\|\cdot\|$ is some suitable metric

norm that combines the sensitivity metric of each set of NDT parameters, $\vec{\psi}$, over the spatial domain $(\Omega)$ and time domain $(\mathrm{I})$. 
By maximizing the above objective function, the total sensitivity of the responses collected by all sensors can be optimized. However, if the candidate sensor and actuator locations are continuously or nearly-continuously distributed throughout a structure, then maximizing Eqn. (4.1) will likely lead to unreasonable layouts of sensors and actuators, specifically in terms of clustering the sensors in small regions. Notghi et al. addressed this issue by developing an additional objective function towards reducing the shared information between different sensors [27]. This concept is also applicable to responses in the time domain, and therefore, a second objective function that quantifies the redundancy of the testing information with respect to NDT parameters can be defined as:

$$
\theta_{i, j}=\arccos \left(\frac{\left\{r^{F E M}\left(\vec{\psi}_{i}, E(\vec{x})\right)\right\} \cdot\left\{r^{F E M}\left(\vec{\psi}_{j}, E(\vec{x})\right)\right\}}{\left\|r^{F E M}\left(\vec{\psi}_{i}, E(\vec{x})\right)\right\|\left\|r^{F E M}\left(\vec{\psi}_{j}, E(\vec{x})\right)\right\|}\right), \quad \forall i, j \in\left[1, N_{T}\right], i \neq j,
$$

where $N_{T}$ is the number of combinations of NDT design parameters, such as locations of sensors and actuators, sensor orientations, excitation frequencies, etc., and $\|\cdot\|$ can be defined such that:

$$
\left\|r^{F E M}\left(\vec{\psi}_{i}, E(\vec{x})\right)\right\|^{2}=\left\{r^{F E M}\left(\vec{\psi}_{i}, E(\vec{x})\right)\right\} \cdot\left\{r^{F E M}\left(\vec{\psi}_{i}, E(\vec{x})\right)\right\} .
$$

Using Eqn. (4.1) and Eqn. (4.2), the optimal NDT design problem can be cast as a multi-objective optimization problem to maximize the sensitivity of the time responses to changes in the material properties, while simultaneously minimizing the redundancy of testing information. Thus, to implement the multi-optimization NDT design problem the two objective functions can be combined in the following form:

$$
\underset{\left\{\vec{\psi}_{k}\right\}_{k=1}^{N_{T}}}{\operatorname{Maximize}}\left\{\begin{array}{l}
C\left(\left\{\vec{\psi}_{k}\right\}_{k=1}^{N_{T}}\right) \\
\operatorname{Minimum}_{\substack{i, j \in\left[1, N_{T}\right] \\
i \neq j}} \theta_{i, j}
\end{array} .\right.
$$

Note that by maximizing the minimum of $\theta_{i, j}$ among all combinations of parameters the above problem is effectively maximizing all values of the redundancy metric to minimize the overall information redundancy of the NDT design. 
One additional note is that the material properties of the structures to be considered, such as the Young's modulus distribution, need to be initialized with some value to solve the multiobjective optimization problem and design the NDT. As described in the previous section, a "healthy" state of the structural properties is typically required and easily estimated as a base state in any damage characterization problem. Therefore, the deviations of material properties in the first objective function (Eqn. (4.1)) can be calculated with respect to this healthy state of the structure. Thus, this optimal NDT design approach is applicable to a wide range of damage and/or material characterization problems. Further small details of the implementation of this design approach for wave propagation-based UT will be discussed in the following case studies. 


\subsection{CASE STUDIES}

The NDT design approach based on maximizing the sensitivity of the NDT response to changes in the material properties while also minimizing information redundancy, was ap-

plied to two simulated case studies to explore the performance, especially for the damage characterization by wave propagation-based UT. Both examples consisted of aluminum plate structures with possible damage represented by a change in stiffness distribution. The first example was a simulated square thin plate which was excited at the center of the surface. The locations and orientations of four sensors were considered as the optimization parameters in the square plate. The second example was a L shape thin plate, and this second example considered the location of excitation along with the locations and orientations of sensors as the NDT design parameters. Note that these example damage characterization problems can also be classified as inverse characterization of the Young's modulus distribution, since the damage herein was assumed to only manifest in the Young's modulus and not result in changes to other material properties, such as density and damping. The design parameters in UT, such as locations of sensors and actuators, were firstly optimized by the proposed design approach. In order to then compare the performance of the Optimal design to a more standard design, a Control UT with uniformly distributed sensors was created in each case study. Then, an inverse characterization was applied to solve for example damage scenarios using both the Optimal design UT and Control UT to shown the capability of the NDT design method to produce solvable inverse problems. 


\subsection{IMPLEMENTATION OF NDT DESIGN AND INVERSE CHARACTERIZATION STRATEGIES}

The optimal NDT design method is potentially applicable to a wide range of inverse characterization problems, including multiple physical properties and NDT types. However, for context the examples discussed herein were specified for damage characterization of structures using wave propagation-based UT. In order to reduce the computational cost associated with this NDT, the structures in both examples were aluminum thin plates, which could be simulated by two-dimensional models based on the plane stress assumption. The UT herein employed the pitch-catch methods, which consisted of a piezoelectric transducer (PZT) as an actuator bonded on the surface of the plate and a set of PZTs as sensors distributed over the plate to measured the transient response of the structures. Gresil et al. have provided a guideline to simulate PZT sensors and actuators [24, 3], and the work herein utilized this basis. The wave excitation of the 4-mm square PZT in the examples was simulated by 12 self-equilibrating forces, as shown in Figure 5.1a, and a 3-cycle tone burst with a center frequency of $50 \mathrm{kHz}$ was used as the excitation signal, as shown in Figure 5.1b. According to the constitutive property of the piezoelectric material, the PZT sensors were simulated by measuring the in-plane strain at the PZT location. The time-varying strain measured by the PZT sensors was directly used to inversely characterize the Young's modulus distribution in the structures. As stated, the damage in the structures was simulated as a semi-localized reduction of the Young's modulus of the structures. Therefore, the sensitivity of the response to changes in the Young's modulus was maximized in Optimal NDT design approach. As stated, the NDT design approach optimized the NDT parameters including the actuator location $\vec{x}_{A}$, as well as the $N_{s}$ sensors locations $\left\{\vec{x}_{S i}\right\}_{i=1}^{N_{S}}$, and sensor orientations $\left\{\vec{\gamma}_{i}\right\}_{i=1}^{N_{S}}$.

In the cases studies, the damage characterization with UT was based on the forward problem described in Chapter 2. Both the experimental data and the simulated transient responses were analyzed using the finite element method based on explicit time integration for dynamic solid mechanics. The healthy (i.e., without Young's modulus reduction) material properties of the aluminum thin plates in both example was defined as $E=69 G P a$, the Poison's ratio was defined as $\nu=0.3$, and the density was defined as $\rho=2700 \mathrm{~kg} / \mathrm{m}^{3}$. In 


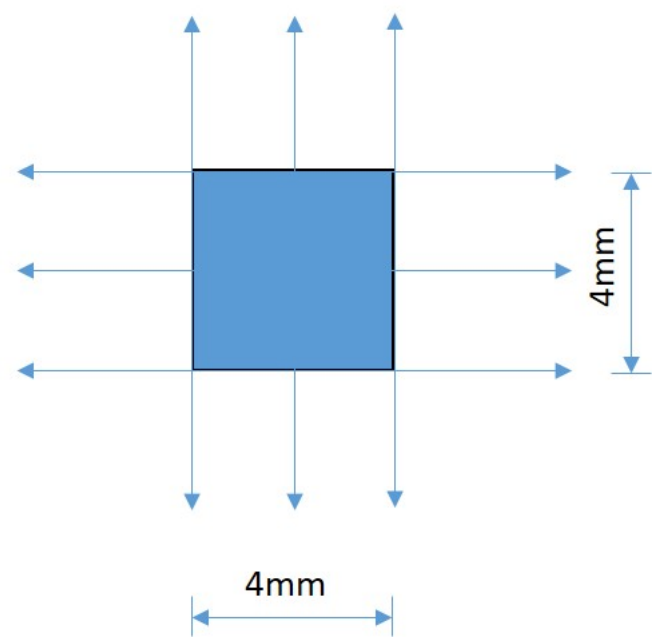

(a)

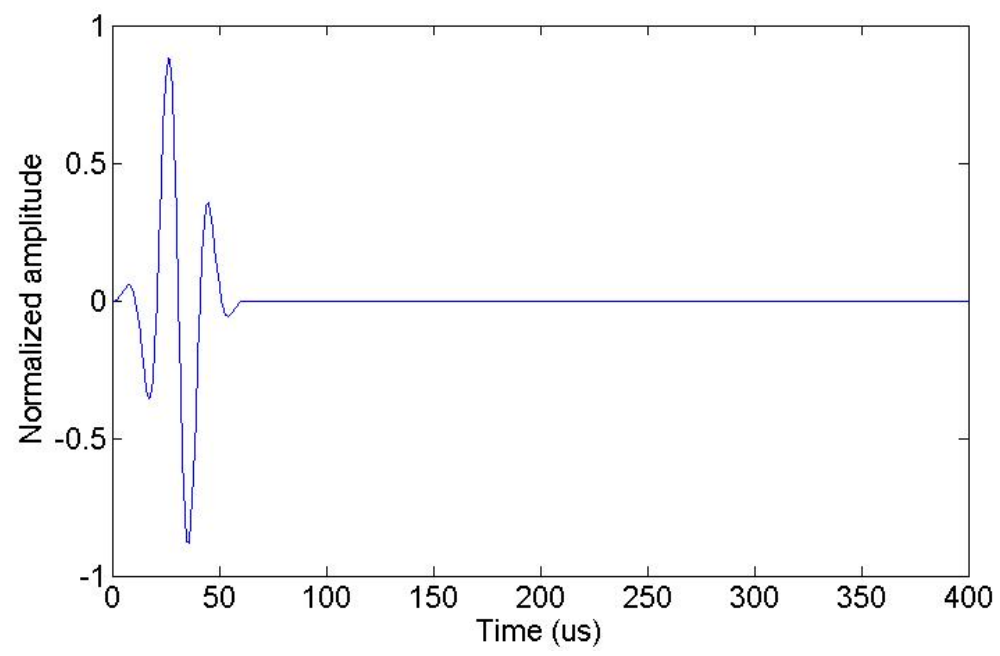

(b)

Figure 5.1: Schematics of the excitation simulated by (a) 12 self-equilibrating forces and (b) the time signal of a 3-cycle tone burst with a center frequency of $50 \mathrm{kHz}$. 
an attempt to add realism for these simulated UT examples, a time shift was assumed to exist between the experimental data (i.e., NDT measurements) and the simulated responses used in the NDE process. In other words, it was assumed that the starting time of the NDT was unknown, as would be the case in real tests, and so the transient responses would need to be aligned in the time domain to make consistent comparison (i.e., to calculate the measurement error) in the inverse solution process. To make these consistent comparisons in time, the time point of the first peak of the transient responses was set to be the initial time $t=0$ of each signal (for the NDT signals and all simulated signals during the NDE process). The signal prior to that first peak were discarded for the damage characterization process, and a window of 200 time steps of the signals following this new zero-time were used for the comparisons. In addition, to represent the noise that would be expected in real experiments, 1\% Gaussian white noise was added into the NDT responses simulated by the FEA, which was implemented as:

$$
r^{E x p}=r_{0}^{E x p}(1+0.01 \Upsilon)
$$

where $r_{0}^{E x p}$ is the original simulated NDT response and $\Upsilon$ is a normally distributed random variable with unit variance and zero mean.

\subsubsection{Optimal Nondestructive Test Design}

As described in Chapter 2, the spatial domain of the structures were discretized into linear trianglular elements, while the time domains were discretized with suitably small fixed time steps, and convergence of all models was thoroughly tested. Utilizing the discretization, the total sensitivity of the NDT responses with respect to the Young's modulus (i.e., the implementation of Eqn. (4.1)) was calculated as:

$$
C=\sum_{i=1}^{N_{S}} \sum_{j=1}^{N_{t}} \sum_{k=1}^{N_{e}} S_{i j k}
$$

where

$$
S_{i j k}=\left|\frac{r^{F E M}\left(t_{j}, \vec{x}_{S i}, \vec{\gamma}_{i}, \vec{x}_{A}, E_{h}\right)-r^{F E M}\left(t_{j}, \vec{x}_{S i}, \vec{\gamma}_{i}, \vec{x}_{A}, E_{D k}\right)}{\frac{1}{2}\left[r^{F E M}\left(t_{j}, \vec{x}_{S i}, \vec{\gamma}_{i}, \vec{x}_{A}, E_{h}\right)+r^{F E M}\left(t_{j}, \vec{x}_{S i}, \vec{\gamma}_{i}, \vec{x}_{A}, E_{D k}\right)\right] \Delta E}\right|
$$


In the above equation, $N_{t}$ is the number of time steps, $N_{e}$ is the number of elements discretizing Young's modulus in space, $E_{h}$ is the Young's modulus distribution without any reduction (i.e., healthy), and $E_{D k}$ is the Young's modulus distribution with a reduction of $\Delta E$ at the $k^{t h}$ element. The sensitivity function $C(E(\vec{x}))$ is the summation of the sensitivity of the measurements of every sensors with respect to Young's modulus reduction located in each element over all time steps. Similarly, the redundancy calculation (i.e., the implementation of Eqn. (4.2)) was defined in the discretized form as:

$$
\theta_{i, j}=\arccos \sum_{k=1}^{N_{t}}\left(\frac{\left\{r^{F E M}\left(t_{k}, \vec{x}_{S i}, \vec{\gamma}_{i}, \vec{x}_{A}, E_{h}\right)\right\} \cdot\left\{r^{F E M}\left(t_{k}, \vec{x}_{S j}, \vec{\gamma}_{j}, \vec{x}_{A}, E_{h}\right)\right\}}{\left\|r^{F E M}\left(t_{k}, \vec{x}_{S i}, \vec{\gamma}_{i}, \vec{x}_{A}, E_{h}\right)\right\|\left\|r^{F E M}\left(t_{k}, \vec{x}_{S i}, \vec{\gamma}_{i}, \vec{x}_{A}, E_{h}\right)\right\|}\right)
$$

Lastly, in order to solve the multi-objective optimization problem described by Eqn. (4.4) to optimally design the NDT, multi-objective Genetic Algorithm (MOGA) [49] was used. This optimization approach produces a set of solutions (rather than a single solution that would be typically obtained with single-objective optimization), that are defined as the set of non-dominated solutions (i.e., none of the solutions have the best value of every objective functions). However, for implementation obviously only one Optimal NDT design solution needs to be selected from the solution set. The nearest to optimal point method for the solution selection was implemented here, which selects the solution with the shortest

distance from a virtual solution that has the "best" values for both objective functions from the solution set.

\subsubsection{Nondestructive Evaluation/Inverse Characterization}

Several inverse characterization tests were performed to determine different Young's modulus distributions from measurement data simulated with the Optimal NDT designs as well as the Control NDT designs to explore the potential improvement in the characterization capabilities with the optimized approach. In order to generate the simulated data the Young's modulus distributions were assumed to be semi-localized in nature. More specifically, the present work utilized a radial basis function $(\mathrm{RBF})$ representation of the semi-localized Young's modulus distribution, as was also used for the representation of damage in thin plates 
in [27]. Thus, the distribution of Young's modulus in the case studies herein was defined by a $\mathrm{RBF}$ representation as:

$$
E(\vec{x})=E_{h}\left[1-\sum_{i=1}^{N_{D}} D_{i} \exp \left(-\frac{\left\|\vec{x}-\vec{\xi}_{i}\right\|^{2}}{c_{i}^{2}}\right)\right]
$$

where $E_{h}$ is the healthy Young's modulus value, $N_{D}$ is the number of RBFs (i.e., damage

regions), $\vec{\xi}_{i}$ is the location of the $i^{t h} \mathrm{RBF}$ center (i.e., damage center), $c_{i}$ is the parameter that controls the breadth of each damage region, $D_{i}$ is the maximum percent reduction in Young's modulus due to each RBF, and $\|\cdot\|$ is the standard $l_{2}$ norm. For simplicity in the following examples the number of damage regions and the healthy modulus were assumed to be known a priori to be one. The inverse characterization approach discussed in Chapter 3 was therefore applied to estimate the four parameters describing the Young's modulus distribution $\left(\xi_{1}, \xi_{2}, c\right.$, and $\left.D\right)$. For the optimization inverse solution process, the objective functional used to quantify the difference between the "experimental" (i.e., simulated experimental) UT response data and the response predicted by the numerical simulation given an estimate of the material parameters (i.e., the implementation of Eqn. (3.1)) was defined as:

$$
J=\frac{1}{N_{t} N_{S}} \sum_{i=1}^{N_{S}} \sum_{j=1}^{N_{t}}\left|\frac{r^{E x p}\left(t_{j}, \vec{x}_{S i}, \vec{\gamma}_{i}, \vec{x}_{A}\right)-r^{F E M}\left(t_{j}, \vec{x}_{S i}, \vec{\gamma}_{i}, \vec{x}_{A}, E(\vec{x})\right)}{\frac{1}{2}\left[r^{E x p}\left(t_{j}, \vec{x}_{S i}, \vec{\gamma}_{i}, \vec{x}_{A}\right)+r^{F E M}\left(t_{j}, \vec{x}_{S i}, \vec{\gamma}_{i}, \vec{x}_{A}, E(\vec{x})\right)\right]}\right|
$$

\subsection{EXAMPLE 1 - SQUARE PLATE}

The first case study consisted of a $1 m \times 1 m \times 0.02 m$ aluminum plate. The bottom boundary of the square plate was assumed to be fixed, while all others boundaries were traction free. In this example, the actuator was assumed to be placed near to the center of the aluminum plate at a location of $X_{A}=0.52 m, Y_{A}=0.52 m$ with respect to the bottom left corner. The actuator location was chosen to be off-center and not considered as a variable in the NDT optimization to help ensure that the Optimal NDT design would be nontrivial for this basic structure geometry. The actuator was simulated to generate a 3-cycle tone burst excitation signal, as shown in Figure 5.2a, with a center frequency of 50kHz. The total duration of the 
simulated UT was taken as $400 \mu s$, and a $1 \mu s$ fixed time increment was used for all FEA, which satisfied the requirements defined in Eqn. (1.4) and Eqn. (2.27). Four strain sensors were assumed to be sufficient to inversely determine the Young's modulus distributions. Therefore, the NDT parameters to be optimized were the coordinates and the orientation (either horizontal or vertical) of the four sensors. An important point is that the coordinates of the sensors were limited as $X_{S i} \in[0.05 m, 0.95 m]$ and $Y_{S i} \in[0.05 m, 0.95 m]$ to reduce the effect from the boundaries, especially the fixed bottom.

After applying the NDT design optimization process, the optimal locations and orientations of four sensors were determined to be three vertically oriented sensors with $X_{S 1}=0.4 \mathrm{~m}$, $Y_{S 1}=0.9 m, X_{S 2}=0.6 m, Y_{S 2}=0.8 m$, and $X_{S 3}=0.9 m, Y_{S 3}=0.2 m$, and one horizontally oriented sensor with $X_{S 4}=0.6 m, Y_{S 4}=0.4 m$. The Control NDT design used for comparison had uniformly distributed sensors with $X_{S 1}=0.25 m, Y_{S 1}=0.25 m, X_{S 2}=0.25 m$, $Y_{S 2}=0.75 m, X_{S 3}=0.75 m, Y_{S 3}=0.75 m$, and $X_{S 4}=0.75 m, Y_{S 4}=0.75 m$, and all were set to measure the vertical strain. Figures $5.2 \mathrm{a}$ and $5.2 \mathrm{~b}$ illustrate the sensor layouts for the Control NDT design and the Optimal NDT design, respectively. In addition, Table. 5.1 shows the values of the sensitivity and redundancy objective functions for both UT NDT designs for this first example. Clearly the sensitivity of the UT with respect to the material properties was significantly improved by the optimal NDT design approach. Alternatively, the information redundancy objective function for the Optimal design and the Control design were relatively similar, which is not necessarily surprising since the values are both relatively high.

Note again that the four sensors were simulated by recording the in-plane strain at all 400 time steps to generate both the simulated experimental data and to predict the response given damage parameters during the inverse solution process. Figure 5.3 shows an example of the raw strain signal (prior to removing the time shift) at location, $X_{S}=0.75 \mathrm{~m}, Y_{S}=0.75 \mathrm{~m}$, with the healthy Young's modulus distribution compared to a Young's modulus distribution with a reduction located at $\epsilon_{1}^{x}=0.65 m, \epsilon_{1}^{y}=0.65 \mathrm{~m}$. It can be observed that the raw transient responses are sensitive to the changes in Young's modulus by comparing the wave signal propagated after damage to the wave signal with healthy material properties. As stated previously, to add realism and eliminate a potential time shift, the sets of raw data 


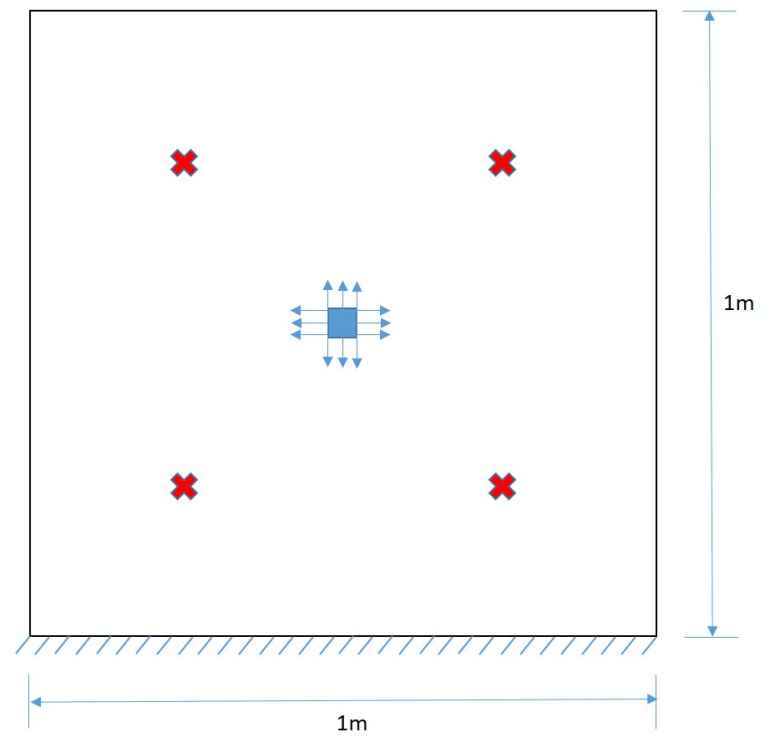

(a)

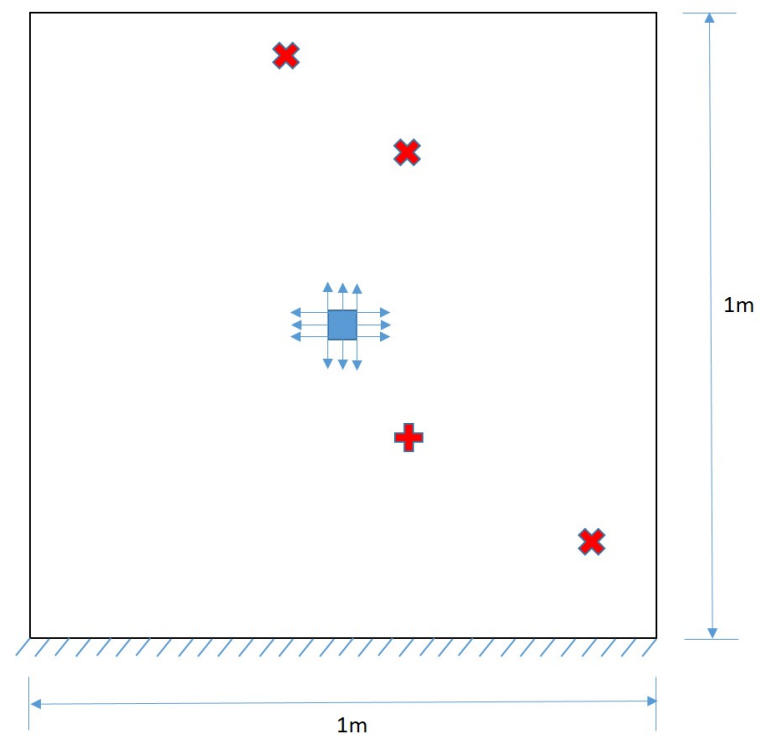

(b)

Figure 5.2: Schematics of (a) the uniformly distributed sensor locations for the Control NDT design and (b) the sensor locations obtained by the Optimal NDT design approach, where "+" refers to the horizontal sensor orientation and " $x$ " refers to the vertical sensor orientation, for Example 1 - Square Plate. 
Table 5.1: Values of the sensitivity objective function (Eqn. (5.3))and the redundancy objective function (Eqn. (5.4)) for Optimal NDT and Control NDT.

\begin{tabular}{lcc}
\hline & Sensitivity performance & Redundancy perfomance \\
\hline Optimal NDT & $3.28 \times 10^{6}$ & $89.99^{\circ}$ \\
\hline Control NDT & $4.88 \times 10^{5}$ & $88.94^{\circ}$ \\
\hline
\end{tabular}

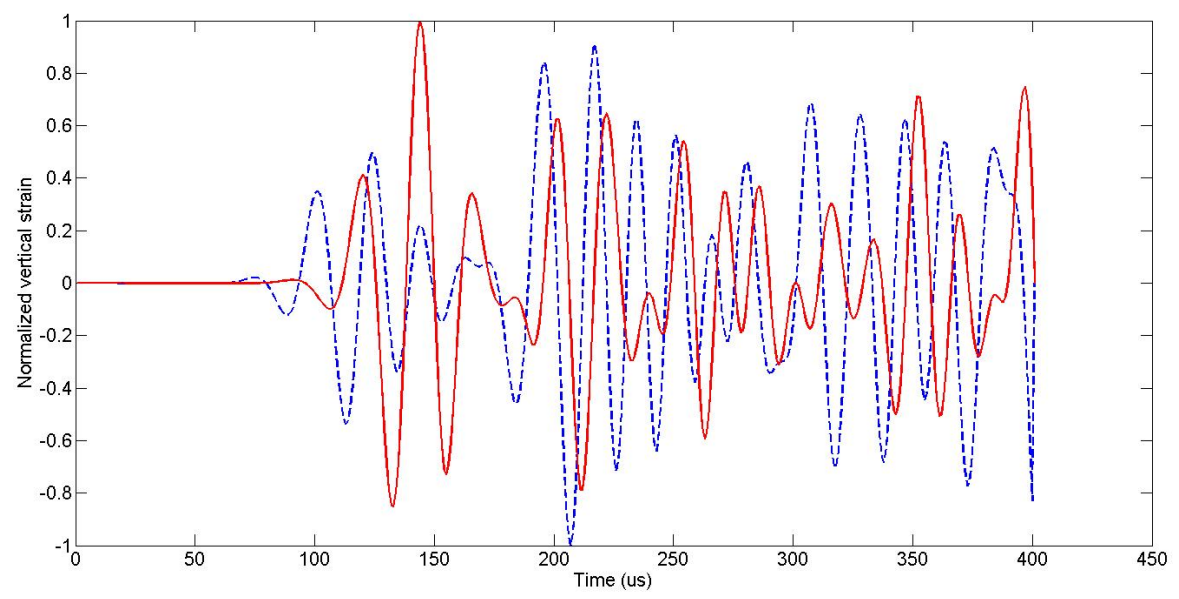

Figure 5.3: Normalized vertical strain signal at location, $X_{S}=0.75 \mathrm{~m}, Y_{S}=0.75 \mathrm{~m}$, with healthy Young's modulus distribution and Young's modulus reduction located at $\epsilon_{1}^{x}=0.65 m$, $\epsilon_{1}^{y}=0.65 m$, where red solid line refers to time signal with Young's modulus reduction and blue dash line refers to time signal with healthy state. 


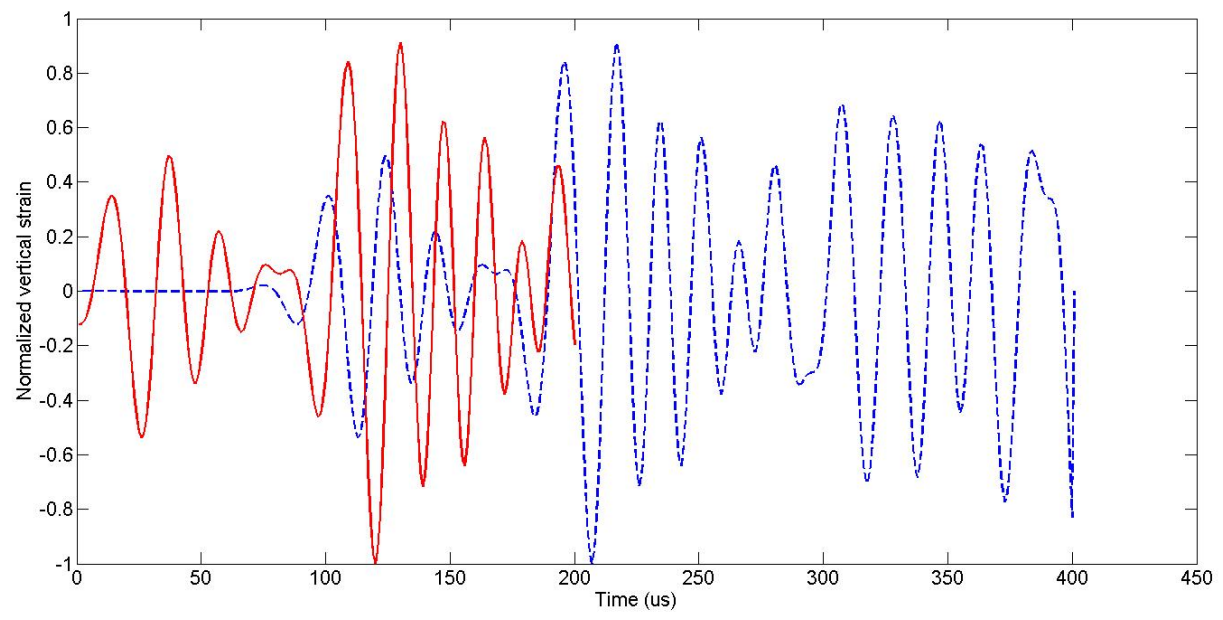

Figure 5.4: Normalized vertical strain signal at location, $X_{S}=0.75 m, Y_{S}=0.75 m$, with healthy Young's modulus distribution before time shift elimination and after time shift elimination where red solid line refers to signal after time shift elimination and blue dash line refers to raw time signal.

were restricted to 200 time steps (i.e., $200 \mu \mathrm{s}$ ) begining with the moment of the first peak of each respective response. Figure 5.4 shows an example of the strain signals before and after this time shift elimination at location, $X_{S}=0.75 m, Y_{S}=0.75 m$, with the healthy Young's modulus distribution.

To test the two NDT designs, four different sets of damage parameters were randomly generated for the plate, and then the inverse characterization process using the SMARS algorithm (introduced in Section 3.1) was applied using the measurement data from each NDT design in turn. The search limits for each damage parameter for the characterization process were set as follows: $\vec{\xi}_{i} \in[0,1] \times[0,1], D_{i} \in[0.1,1]$ and $c_{i} \in[0,0.1]$. The stopping criteria for the SMARS algorithm was set to 400 finite element analyses for each inverse characterization trial. Figures 5.5, 5.6, 5.7, and 5.8 show the Target (i.e., simulated experimental) Young's modulus distribution, the distribution inversely characterized by the Optimal NDT design, and the distribution inversely characterized by the Control NDT design for this first 


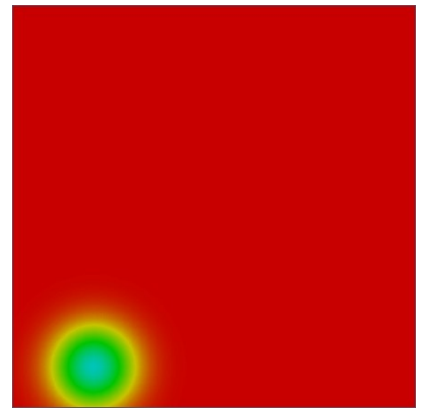

(a)

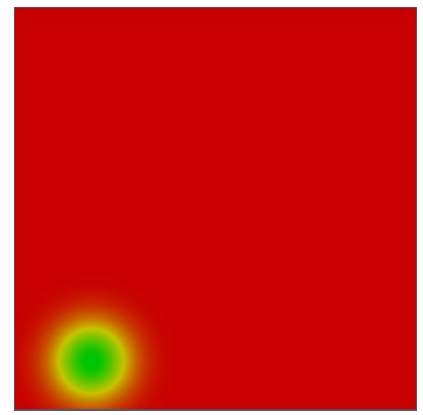

(b)

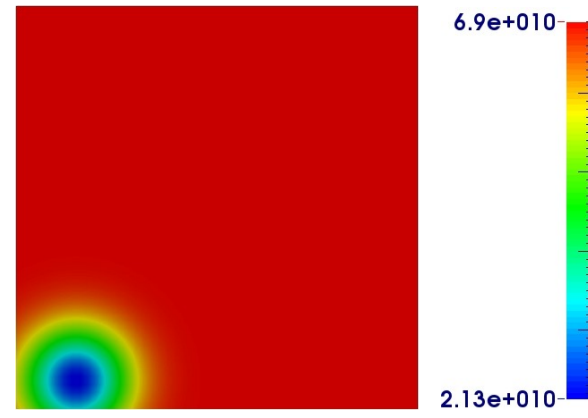

(c)

Figure 5.5: Spatial Young's modulus distribution of (a) Target (b) inverse characterization by Optimal NDT design (c) inverse characterization by Control NDT design for Example 1 - Square Plate with damage location (1).

example for the four different sets of damage parameters, respectively. In order to quantify and better compare the inverse solutions from both the Control and Optimal NDT designs, Tables 5.2, 5.3, 5.4 and 5.5 show the Target values for the damage parameters compared to the inverse solutions for each NDT design, as well as the relative $L_{2}$-Error and the relative $L_{\infty}$-Error for the estimated Young's modulus distributions for the four damage parameter cases, respectively. Moreover, the mean and standard deviation of the relative $L_{2}$-Error and the relative $L_{\infty}$-Error for the estimated Young's modulus distributions for the four different damage parameter trials is shown in Table 5.6.

As seen in the comparisons, the inverse characterization of the Young's modulus distribution from the Optimal NDT were overall substantially more accurate than that estimated by the Control NDT. Both the Optimal and the Control UT were able to provide relatively accurate inverse characterization of the Young's modulus. However, the Optimal NDT provided a consistently more accurate solution with nearly $50 \%$ less in mean error compared to the Control NDT. Although the Optimal and the Control NDT had a relatively close $L_{2^{-}}$ Error and $L_{\infty}$-Error with respect to two of the damage parameter trials (Target(2) and (4)), the estimates of the damage locations by the Optimal NDT were still significantly better for those cases. 


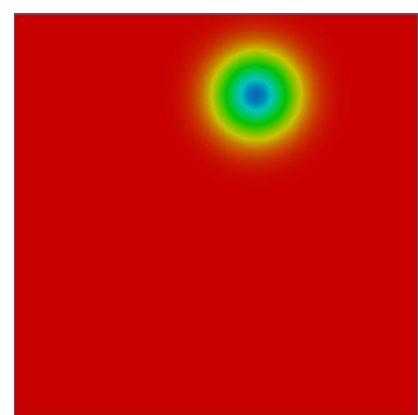

(a)

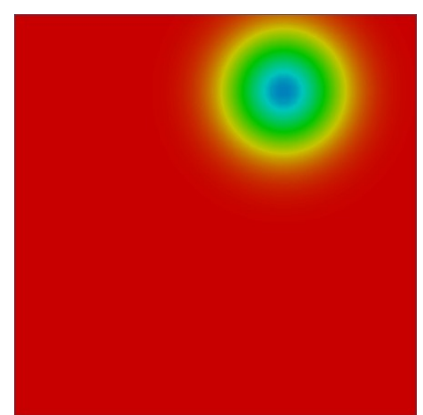

(b)

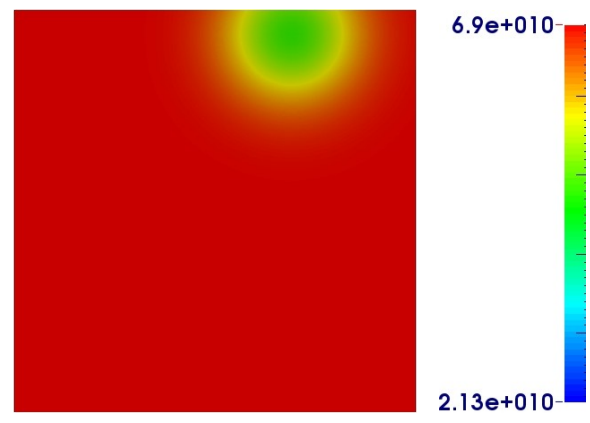

(c)

Figure 5.6: Spatial Young's modulus distribution of (a) Target (b) inverse characterization by Optimal NDT design (c) inverse characterization by Control NDT design for Example 1 - Square Plate with damage location (2).

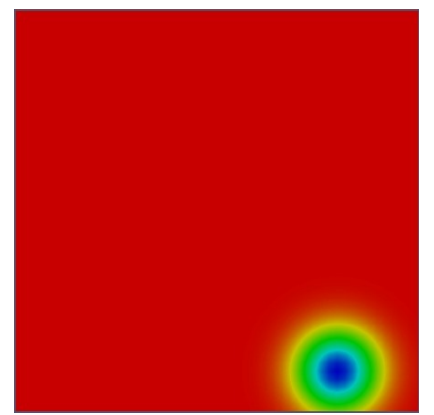

(a)

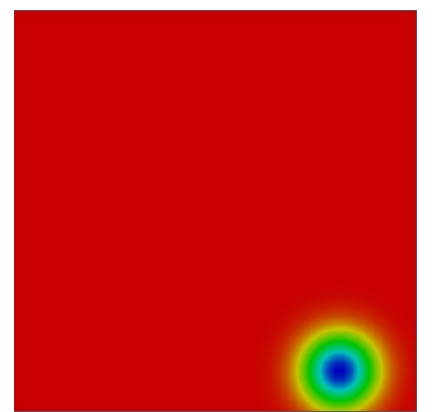

(b)

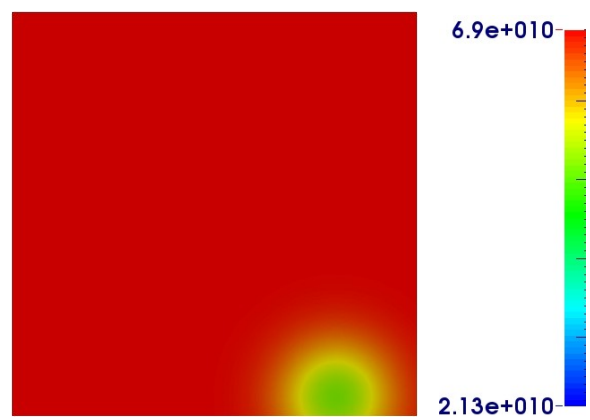

(c)

Figure 5.7: Spatial Young's modulus distribution of (a) Target (b) inverse characterization by Optimal NDT design (c) inverse characterization by Control NDT design for Example 1 - Square Plate with damage location (3). 


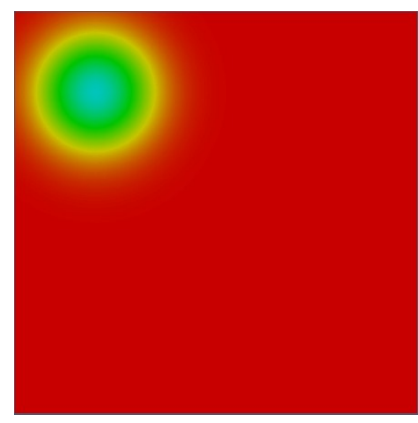

(a)

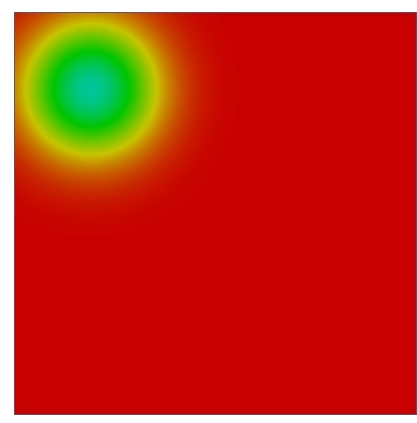

(b)

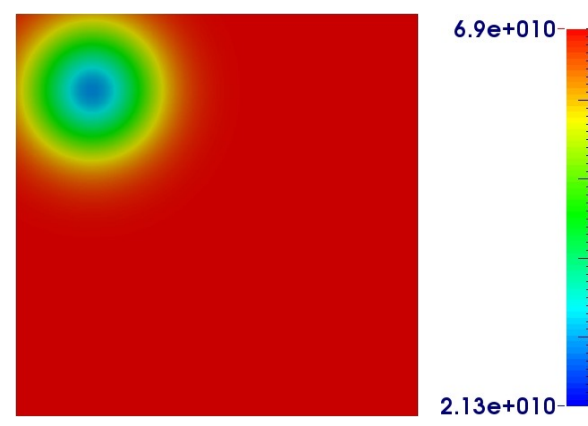

(c)

Figure 5.8: Spatial Young's modulus distribution of (a) Target (b) inverse characterization by Optimal NDT design (c) inverse characterization by Control NDT design for Example 1 - Square Plate with damage location (4).

Table 5.2: The solution and the relative $L_{2}$-Error and the relative $L_{\infty}$-Error of the Young's modulus distribution with respect to the expected results estimated by the inverse characterization process in the Optimal NDT design and the Control NDT design for Example 1 Square Plate with damage location (1).

\begin{tabular}{ccccccc}
\hline & $c_{1}$ & $D_{1}$ & $\epsilon_{1}^{x}$ & $\epsilon_{1}^{y}$ & $L_{2}$-Error & $L_{\infty}$-Error \\
\hline Target Value (1) & 0.010 & 0.60 & 0.20 & 0.10 & - & - \\
\hline $\begin{array}{c}\text { Optimal NDT Design } \\
\text { Solution }\end{array}$ & 0.009 & 0.46 & 0.19 & 0.12 & 0.025 & 0.017 \\
\hline $\begin{array}{c}\text { Control NDT Design } \\
\text { Solution }\end{array}$ & 0.017 & 0.70 & 0.15 & 0.07 & 0.055 & 0.550 \\
\hline
\end{tabular}


Table 5.3: The solution and the relative $L_{2}$-Error and the relative $L_{\infty}$-Error of the Young's modulus distribution with respect to the expected results estimated by the inverse characterization process in the Optimal NDT design and the Control NDT design for Example 1 Square Plate with damage location (2).

\begin{tabular}{cccccccc}
\hline & $c_{1}$ & $D_{1}$ & $\epsilon_{1}^{x}$ & $\epsilon_{1}^{y}$ & $L_{2}$-Error & $L_{\infty}$-Error \\
\hline Target Value (2) & 0.010 & 0.70 & 0.60 & 0.80 & - & - \\
\hline $\begin{array}{c}\text { Optimal NDT Design } \\
\text { Solution }\end{array}$ & 0.020 & 0.74 & 0.67 & 0.81 & 0.081 & 0.490 \\
\hline $\begin{array}{c}\text { Control NDT Design } \\
\text { Solution }\end{array}$ & 0.028 & 0.31 & 0.69 & 0.94 & 0.090 & 0.293 \\
\hline
\end{tabular}

Table 5.4: The solution and the relative $L_{2}$-Error and the relative $L_{\infty}$-Error of the Young's modulus distribution with respect to the expected results estimated by the inverse characterization process in the Optimal NDT design and the Control NDT design for Example 1 Square Plate with damage location (3).

\begin{tabular}{ccccccc}
\hline & $c_{1}$ & $D_{1}$ & $\epsilon_{1}^{x}$ & $\epsilon_{1}^{y}$ & $L_{2}$-Error & $L_{\infty}$-Error \\
\hline Target Value (3) & 0.010 & 0.80 & 0.80 & 0.10 & - & - \\
\hline $\begin{array}{c}\text { Optimal NDT Design } \\
\text { Solution }\end{array}$ & 0.008 & 0.89 & 0.81 & 0.10 & 0.015 & 0.116 \\
\hline $\begin{array}{c}\text { Control NDT Design } \\
\text { Solution }\end{array}$ & 0.020 & 0.30 & 0.80 & 0.05 & 0.060 & 0.062 \\
\hline
\end{tabular}


Table 5.5: The solution and the relative $L_{2}$-Error and the relative $L_{\infty}$-Error of the Young's modulus distribution with respect to the expected results estimated by the inverse characterization process in the Optimal NDT design and the Control NDT design for Example 1 Square Plate with one damage location (4).

\begin{tabular}{cccccccc}
\hline & $c_{1}$ & $D_{1}$ & $\epsilon_{1}^{x}$ & $\epsilon_{1}^{y}$ & $L_{2}$-Error & $L_{\infty}$-Error \\
\hline Target Value (4) & 0.020 & 0.60 & 0.20 & 0.80 & - & - \\
\hline $\begin{array}{c}\text { Optimal NDT Design } \\
\text { Solution }\end{array}$ & 0.026 & 0.62 & 0.19 & 0.81 & 0.025 & 0.11 \\
\hline $\begin{array}{c}\text { Control NDT Design } \\
\text { Solution }\end{array}$ & 0.025 & 0.59 & 0.19 & 0.81 & 0.020 & 0.09 \\
\hline
\end{tabular}

Table 5.6: Mean and standard deviation of the relative $L_{2}$-Error and the relative $L_{\infty}$-Error of the Young's modulus distribution for four sets of damage parameters.

\begin{tabular}{ccc}
\hline & $L_{2}$-Error & $L_{\infty}$-Error \\
\hline Optimal NDT Design & & \\
Mean & 0.037 & 0.183 \\
Std. Dev. & 0.030 & 0.210 \\
\hline Control NDT Design & & \\
Mean & 0.056 & 0.264 \\
Std. Dev. & 0.029 & 0.226 \\
\hline
\end{tabular}




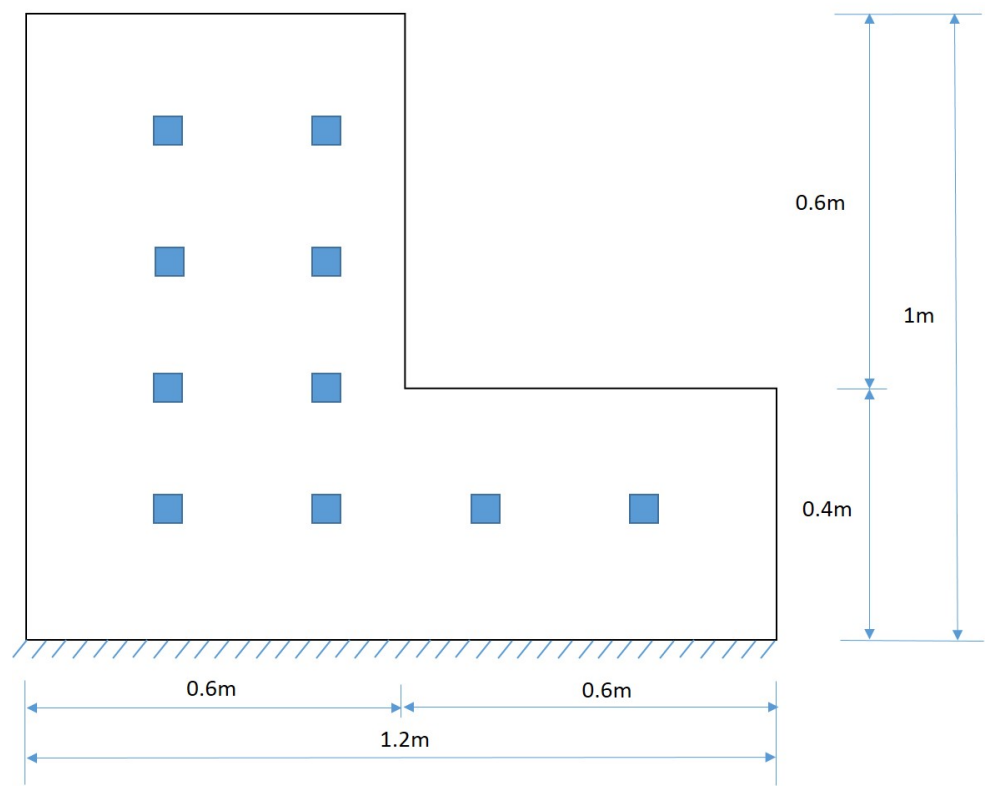

Figure 5.9: Schematic of Example 2 - L Shaped Plate and the potential actuator locations for the Optimal NDT design process.

\subsection{EXAMPLE 2 - L SHAPED PLATE}

To examine how the optimal NDT design approach would generalize, the second case study considered a more complex structure than the first example, and consisted of a L shaped aluminum plate with outer dimensions of $1.2 \mathrm{~m} \times 1 \mathrm{~m}$ and a thickness of $0.02 \mathrm{~m}$, as shown in Figure 5.9. The $\mathrm{L}$ shaped plate was taken to be fixed at the bottom with the remaining boundaries traction free. The actuator and sensors were simulated using the same method as in the first square plate example. The total duration of the simulated UT was taken as $400 \mu s$, and a $0.25 \mu s$ fixed time increment was used for all FEA. A smaller time increment was required in this example because of the more complex geometry than the first example. However, to reduce the computational expense, the sensor data from the FEA was downsampled to every $1 \mu \mathrm{s}$. For this second example the location of actuator was considered as an optimization parameter with 10 discrete candidate locations, as shown in Figure 5.9. Again, four strain sensors were assumed to be sufficient to inversely determine the Young's modulus 
distributions. Therefore, the NDT parameters to be optimized for this second example were the actuator location and the coordinates and the orientation (either horizontal or vertical) of the four sensors. The potential locations of the sensors were restricted to be at least $0.1 \mathrm{~m}$ away from the fixed bottom boundary and at least $0.05 \mathrm{~m}$ away from the other boundaries.

After applying the NDT design optimization process, the optimal location of the actuator was determined to be $X_{A}=0.24 m, Y_{A}=0.6 m$, and the optimal locations and orientations of four sensors were determined to be three vertically oriented sensors with $X_{S 1}=0.1 \mathrm{~m}, Y_{S 1}=$ $0.9 m, X_{S 2}=0.2 m, Y_{S 2}=0.7 m$, and $X_{S 3}=1.0 m, Y_{S 3}=0.2 m$, and one horizontally oriented sensor with $X_{S 4}=0.1 m, Y_{S 4}=0.2 m$. The Control NDT design used for comparison again had regularly distributed sensors all measuring vertical strain with $X_{S 1}=0.2 m, Y_{S 1}=0.8 m$, $X_{S 2}=0.2 m, Y_{S 2}=0.4 m, X_{S 3}=0.4 m, Y_{S 3}=0.2 m$, and $X_{S 4}=0.8 m, Y_{S 4}=0.2 m$. Figures 5.10a and 5.10b show the sensor and actuator layouts for the Control NDT design and the Optimal NDT design, respectively. In addition, Table 5.7 shows the values of the sensitivity and redundancy objective functions for both UT NDT designs for this second example. Similar as before, the improvement of the sensitivity of the system responses to changes in Young's modulus for the Optimal NDT design was significant with a nearly twentyfold increase in the sensitivity metric compared to the Control NDT design. This substantial improvement in the NDT design is reasonable, largely due to the increased complexity of the structure and the added benefit of designing the actuator location as well. Again, the redundancy metrics of both the Optimal NDT design and the Control NDT design were relatively high, nearly reaching the maximum possible value of $90^{\circ}$.

As before, the potential time shift was eliminated from all response measurements and a window of $200 \mu s$ of each signal was utilized for the inverse process. Again, to test the performance of the Optimal NDT design compared to the Control NDT design for damage characterization for the L shaped plate, four different sets of damage parameters were randomly generated for the plate, and then the inverse characterization process using the SMARS algorithm was applied using the measurement data from each NDT design in turn. Due to the increased complexity of this second example, the stopping criteria for the SMARS algorithm was increased to 600 finite element analyses for each inverse characterization trial. Figures 5.11,5.12,5.13, and 5.14 show the Target Young's modulus 


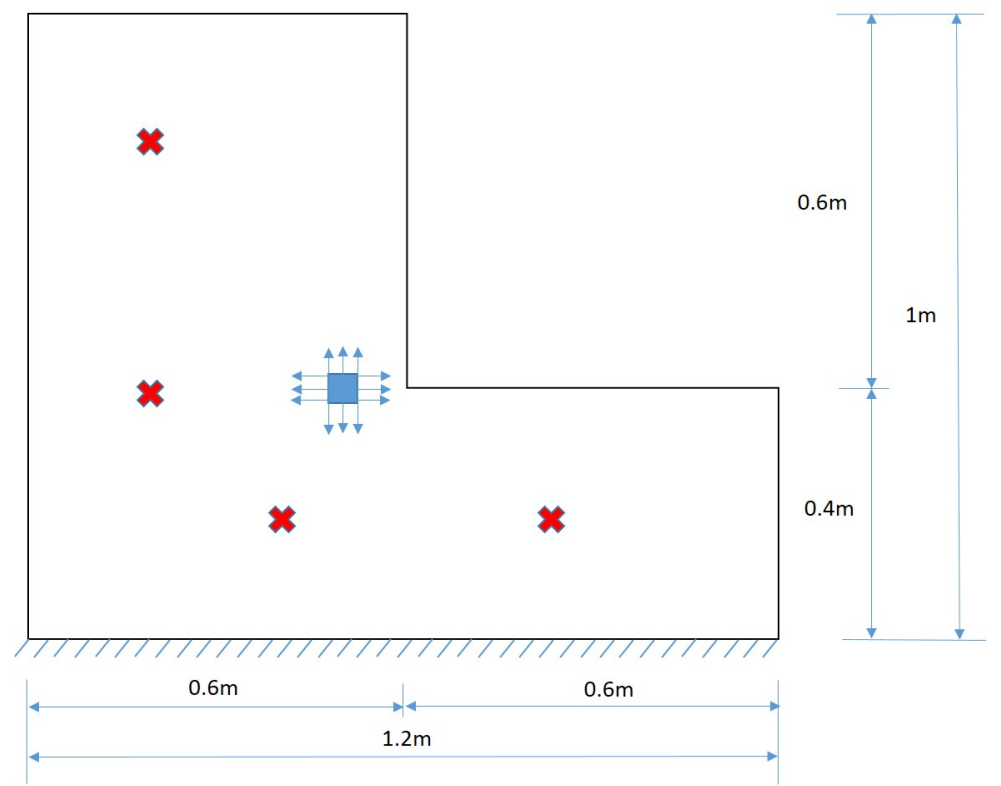

(a)

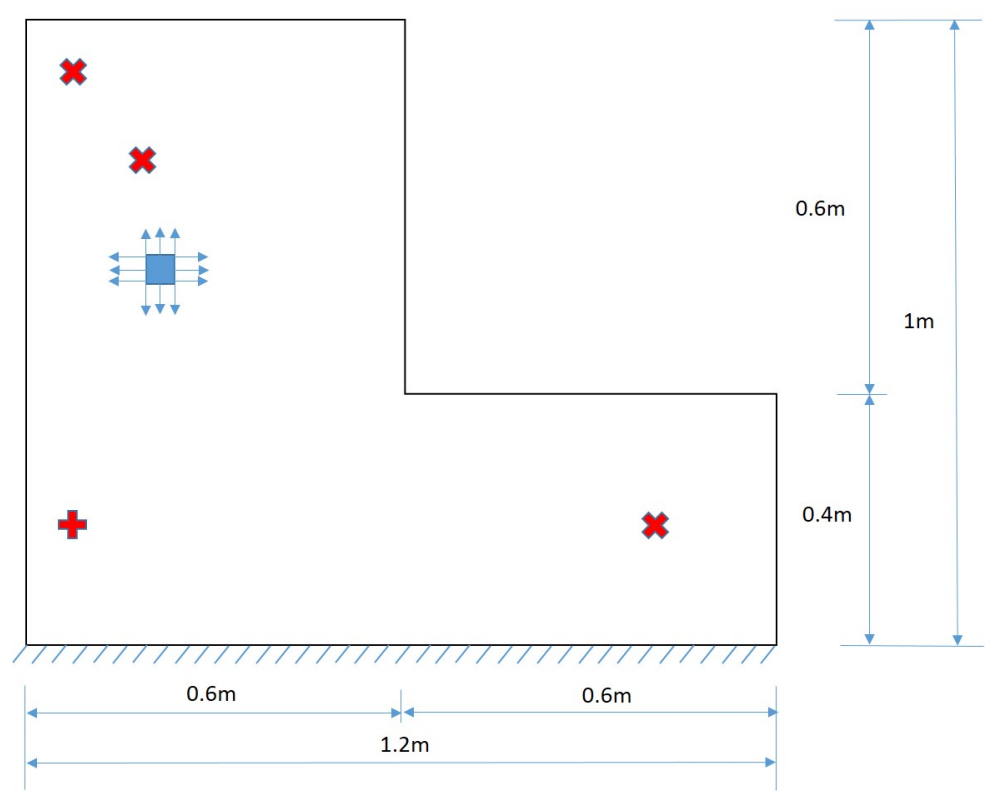

(b)

Figure 5.10: Schematic of (a) the regularly distributed sensor and actuator locations for the Control design and (b) the sensor and actuator locations obtained for the Optimal NDT design, where "+" refers to the horizontal sensor orientation and " $x$ " refers to the vertical sensor orientation, for Example 2 - L Shaped Plate. 
Table 5.7: Values of the sensitivity objective function (Eqn. (5.3))and the redundancy objective function (Eqn. (5.4)) for Optimal NDT and Control NDT.

\begin{tabular}{lcc}
\hline & Sensitivity performance & Redundancy perfomance \\
\hline Optimal NDT & $5.07 \times 10^{6}$ & $89.99^{\circ}$ \\
\hline Control NDT & $3.02 \times 10^{5}$ & $89.98^{\circ}$ \\
\hline
\end{tabular}

distribution, the distribution inversely characterized by the Optimal NDT design, and the distribution inversely characterized by the Control NDT design for this second example for the four different sets of damage parameters, respectively. To further compare the inverse solutions from both the Control and Optimal NDT designs, Tables 5.8, 5.9, 5.10, and 5.11 show the Target values for the damage parameters compared to the inverse solutions for each NDT design, as well as the relative $L_{2}$-Error and the relative $L_{\infty}$-Error for the estimated Young's modulus distributions for the four damage parameter cases, respectively. Lastly, Table 5.12 shows the mean and standard deviation of the relative $L_{2}$-Error and the relative $L_{\infty}$-Error for the estimated Young's modulus distributions for the four different damage parameter trials. Although the total number of finite element analyses increased to 600 for this second example, the overall accuracy of inverse characterization of the Young's modulus with both the Optimal NDT design and the Control NDT design decreased. However, similar to the first example, the distribution of Young's modulus estimated by the Optimal NDT design remained consistently more accurate than the Control NDT design with a mean $L_{2^{-}}$ error near to $6 \%$ for the Optimal NDT design compared to $10 \%$ for the Control. Moreover, since the difficulty of the damage characterization problem increased in this second example, the Optimal NDT design improved the accuracy of the inverse characterization results even more significantly than was seen in the first example. In particular, the capability of the Optimal NDT design to determine the damage location was shown to be substantially better than the Control NDT design, while the results of the Control NDT design also overestimated the breadth of the damage $c_{1}$ for all four sets of assumed damage parameters in comparison 


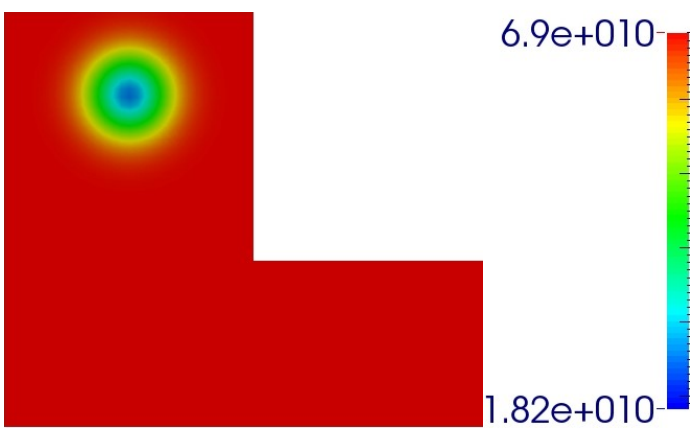

(a)

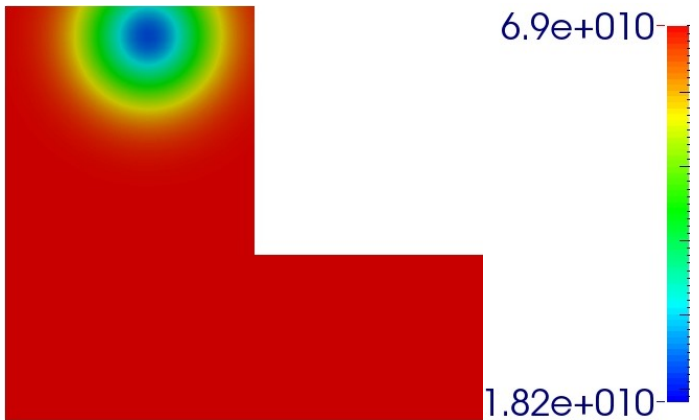

(b)

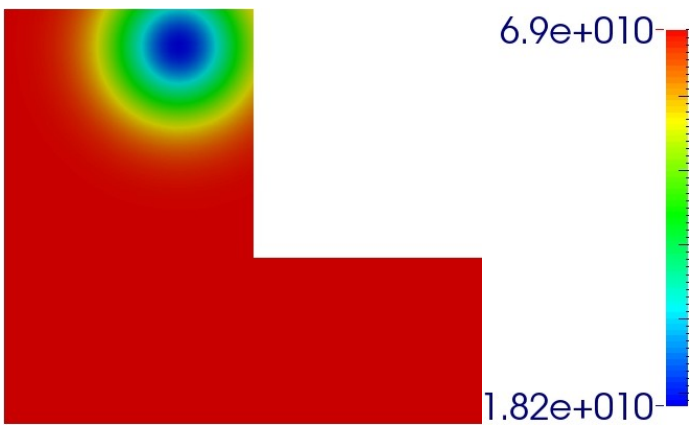

(c)

Figure 5.11: Spatial Young's modulus distribution of (a) Target (b) inverse characterization by Optimal NDT design (c) inverse characterization by Control NDT design for Example 2 - L shape Plate with damage parameters (1). 


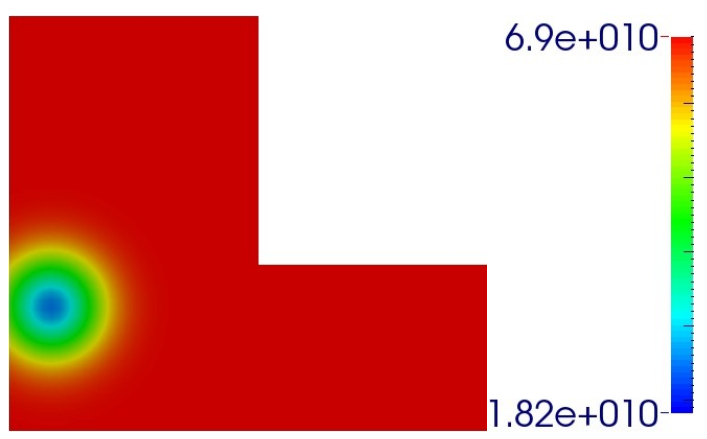

(a)

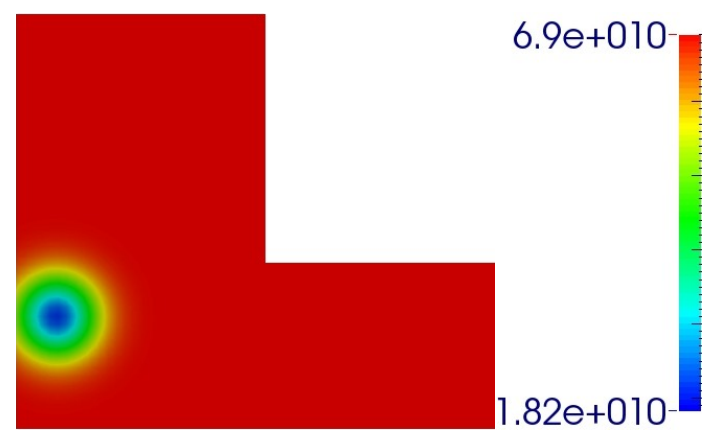

(b)

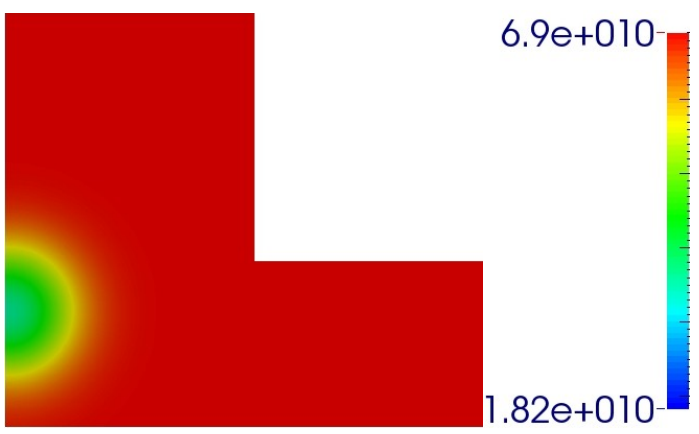

(c)

Figure 5.12: Spatial Young's modulus distribution of (a) Target (b) inverse characterization by Optimal NDT design (c) inverse characterization by Control NDT design for Example 2 - L shape Plate with damage parameters (2). 


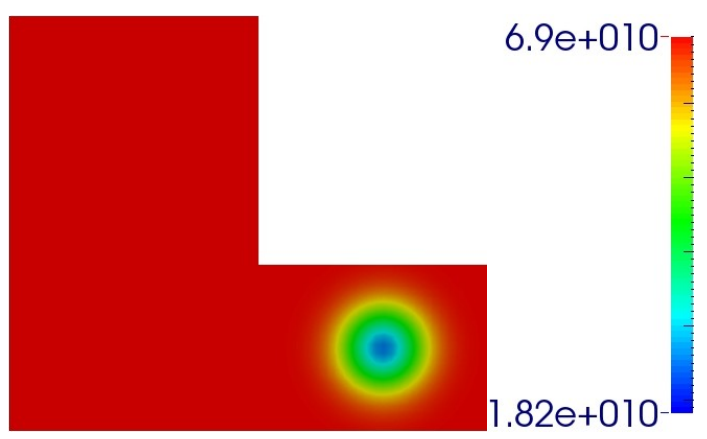

(a)

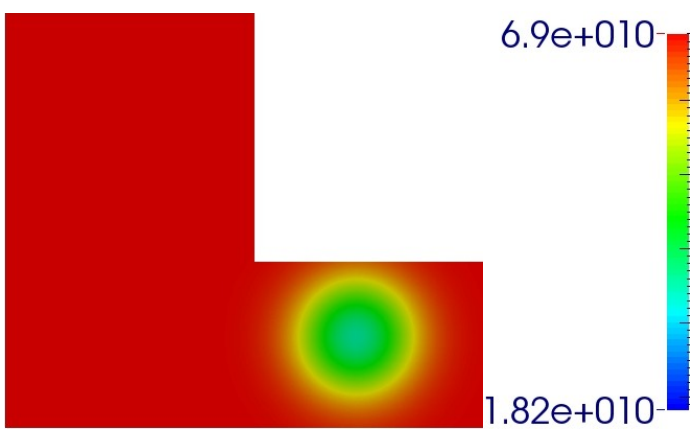

(b)

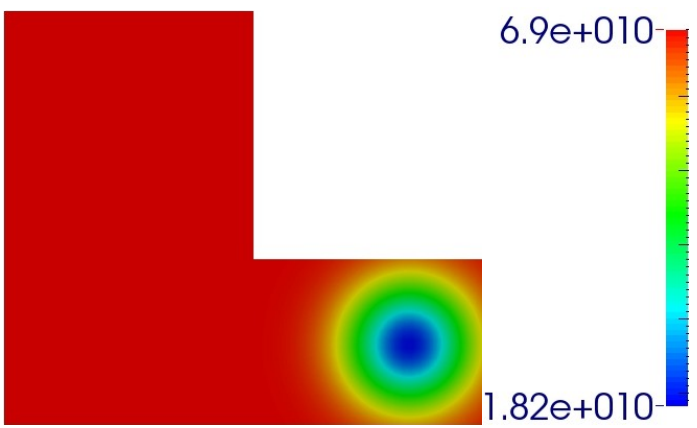

(c)

Figure 5.13: Spatial Young's modulus distribution of (a) Target (b) inverse characterization by Optimal NDT design (c) inverse characterization by Control NDT design for Example 2 - L shape Plate with damage parameters (3). 


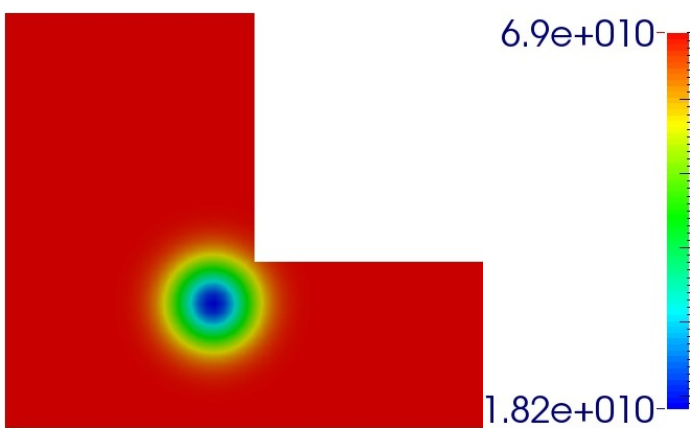

(a)

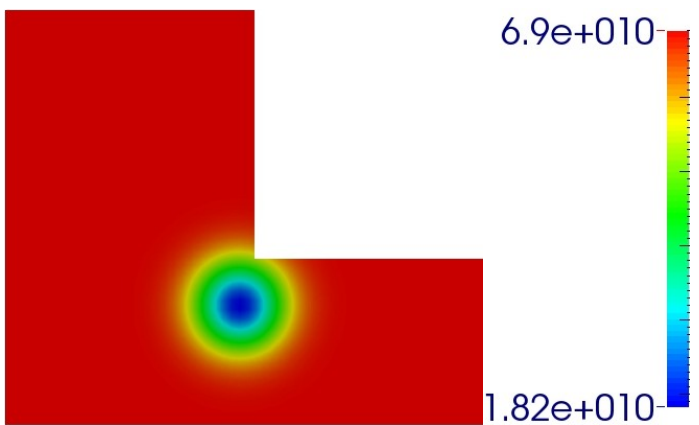

(b)

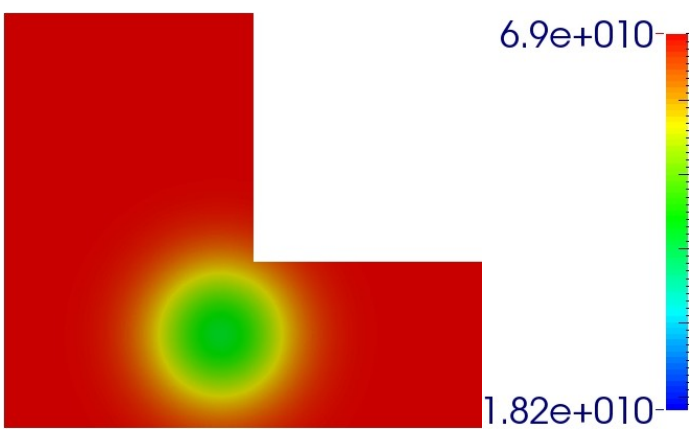

(c)

Figure 5.14: Spatial Young's modulus distribution of (a) Target (b) inverse characterization by Optimal NDT design (c) inverse characterization by Control NDT design for Example 2 - L shape Plate with damage parameters (4). 
Table 5.8: The solution and the relative $L_{2}$-Error and the relative $L_{\infty}$-Error of the Young's modulus distribution with respect to the expected results estimated by the inverse characterization process in the Optimal NDT design and the Control NDT design for Example 2 $\mathrm{L}$ shape plate with damage parameters (1).

\begin{tabular}{ccccccc}
\hline & $c_{1}$ & $D_{1}$ & $\epsilon_{1}^{x}$ & $\epsilon_{1}^{y}$ & $L_{2}$-Error & $L_{\infty}$-Error \\
\hline Target Value (1) & 0.010 & 0.70 & 0.30 & 0.80 & - & - \\
\hline $\begin{array}{c}\text { Optimal NDT Design } \\
\text { Solution }\end{array}$ & 0.024 & 0.68 & 0.34 & 0.92 & 0.084 & 0.47 \\
\hline $\begin{array}{c}\text { Control NDT Design } \\
\text { Solution }\end{array}$ & 0.028 & 0.81 & 0.42 & 0.91 & 0.153 & 0.700 \\
\hline
\end{tabular}

Table 5.9: The solution and the relative $L_{2}$-Error and the relative $L_{\infty}$-Error of the Young's modulus distribution with respect to the expected results estimated by the inverse characterization process in the Optimal NDT design and the Control NDT design for Example 2 L shape Plate with damage parameters (2).

\begin{tabular}{ccccccc}
\hline & $c_{1}$ & $D_{1}$ & $\epsilon_{1}^{x}$ & $\epsilon_{1}^{y}$ & $L_{2}$-Error & $L_{\infty}$-Error \\
\hline Target Value (2) & 0.015 & 0.70 & 0.10 & 0.30 & - & - \\
\hline $\begin{array}{c}\text { Optimal NDT Design } \\
\text { Solution }\end{array}$ & 0.010 & 0.70 & 0.10 & 0.27 & 0.041 & 0.227 \\
\hline $\begin{array}{c}\text { Control NDT Design } \\
\text { Solution }\end{array}$ & 0.025 & 0.54 & 0.007 & 0.28 & 0.060 & 0.321 \\
\hline
\end{tabular}


Table 5.10: The solution and the relative $L_{2}$-Error and the relative $L_{\infty}$-Error of the Young's modulus distribution with respect to the expected results estimated by the inverse characterization process in the Optimal NDT design and the Control NDT design for Example 2 L shape Plate with damage parameters (3).

\begin{tabular}{ccccccc}
\hline & $c_{1}$ & $D_{1}$ & $\epsilon_{1}^{x}$ & $\epsilon_{1}^{y}$ & $L_{2}$-Error & $L_{\infty}$-Error \\
\hline Target Value (3) & 0.010 & 0.70 & 0.90 & 0.20 & - & - \\
\hline $\begin{array}{c}\text { Optimal NDT Design } \\
\text { Solution }\end{array}$ & 0.019 & 0.50 & 0.85 & 0.22 & 0.062 & 0.275 \\
\hline $\begin{array}{c}\text { Control NDT Design } \\
\text { Solution }\end{array}$ & 0.023 & 0.80 & 0.98 & 0.19 & 0.125 & 0.522 \\
\hline
\end{tabular}

Table 5.11: The solution and the relative $L_{2}$-Error and the relative $L_{\infty}$-Error of the Young's modulus distribution with respect to the expected results estimated by the inverse characterization process in the Optimal NDT design and the Control NDT design for Example 2 L shape Plate with one damage parameters (4).

\begin{tabular}{ccccccc}
\hline & $c_{1}$ & $D_{1}$ & $\epsilon_{1}^{x}$ & $\epsilon_{1}^{y}$ & $L_{2}$-Error & $L_{\infty}$-Error \\
\hline Target Value (4) & 0.010 & 0.80 & 0.50 & 0.30 & - & - \\
\hline $\begin{array}{c}\text { Optimal NDT Design } \\
\text { Solution }\end{array}$ & 0.012 & 0.74 & 0.56 & 0.29 & 0.077 & 0.362 \\
\hline $\begin{array}{c}\text { Control NDT Design } \\
\text { Solution }\end{array}$ & 0.029 & 0.44 & 0.52 & 0.22 & 0.090 & 0.438 \\
\hline
\end{tabular}


Table 5.12: Mean and standard deviation of the relative $L_{2}$-Error and the relative $L_{\infty}$-Error of the Young's modulus distribution for four damage locations.

\begin{tabular}{ccc}
\hline & $L_{2}$-Error & $L_{\infty}$-Error \\
\hline Optimal NDT Design & & \\
Mean & 0.066 & 0.334 \\
Std. Dev. & 0.019 & 0.107 \\
\hline Control NDT Design & & \\
Mean & 0.107 & 0.495 \\
Std. Dev. & 0.041 & 0.160 \\
\hline
\end{tabular}

to the Optimal NDT design. Overall, the Optimal NDT design for wave propagation-based NDT was shown to provide almost $60 \%$ more accurate inverse solutions for the Young's modulus distributions compared to the estimates using the Control NDT design. 


\subsection{CONCLUSIONS AND FUTURE DIRECTIONS}

An optimal NDT design approach for wave propagation-based NDT for damage characterization and/or inverse characterization of material properties was presented. The optimal NDT design approach is based on the concept of maximizing the sensitivity of the NDT measurements to changes in the physical properties of the structure along with an additional design objective to minimize the test responses redundancy. This multi-objective optimization design approach was specifically outlined in the context of wave propagation-based NDT, such as ultrasonic testing. Two simulated case studies of damage characterization with UT were presented to evaluate the capability of the proposed NDT design method to provide NDT designs that can be used to accurately characterize damage and/or material properties in structures. Through the cases studies, the capability of the optimal NDT design method to improve wave propagation-based NDT by providing significantly higher response sensitivities and lower information redundancy was shown. Furthermore, comparing to more standard "control" NDT designs, the optimal test method was shown to provide testing information that led to substantially more accurate and stable results for inverse material characterization problems.

The potential was shown for the proposed optimal NDT design method to improve the solvability of inverse problems in basic structures subject to dynamic testing. However,

further work is needed to study the performance of this NDT design approach in more complex wave propagation-based NDT systems, which may require multiple actuators and more sensors or have multiple damage locations. In addition, the attenuation of the ultrasonic wave should be considered in future work as this could have a substantial effect on the resulting optimal NDT designs and achievable characterization capabilities. 


\section{BIBLIOGRAPHY}

[1] Don E Bray and Don McBride. Nondestructive testing techniques. NASA STI/Recon Technical Report A, 93:17573, 1992.

[2] Ajay Raghavan and Carlos ES Cesnik. Review of guided-wave structural health monitoring. Shock and Vibration Digest, 39:91-116, 2007.

[3] M Gresil, V Giurgiutiu, Y Shen, and B Poddar. Guidelines for using the finite element method for modeling guided lamb wave propagation in shm processes. In 6th European Workshop on Structural Health Monitoring, pages 3-6, 2012.

[4] Miguel a Aguiló, Wilkins Aquino, John C Brigham, and Mostafa Fatemi. An inverse problem approach for elasticity imaging through vibroacoustics. IEEE transactions on medical imaging, 29(4):1012-21, April 2010.

[5] Wilkins Aquino and John C. Brigham. Self-learning finite elements for inverse estimation of thermal constitutive models. International Journal of Heat and Mass Transfer, 49(1516):2466-2478, July 2006.

[6] K. Atchonouglo, M. Banna, C. Vallée, and J. C. Dupré. Inverse transient heat conduction problems and identification of thermal parameters. Heat and Mass Transfer, 45(1):2329, April 2008.

[7] M. Chierichetti and M. Ruzzene. Dynamic displacement field reconstruction through a limited set of measurements: Application to plates. Journal of Sound and Vibration, 331(21):4713-4728, October 2012.

[8] MT Martin. Inverse problems in structural dynamics. pages 2-5, 1994.

[9] A. Rytter and Poul Henning Kirkegaard. Vibration Based Inspection of Civil Engineering Structures. Aalborg Universitetsforlag, 1994.

[10] S.K. Thyagarajan, M.J. Schulz, P.F. Pai, and J. Chung. Detecting structural damage using frequency response functions. Journal of Sound and Vibration, 210(1):162 - 170, 1998. 
[11] Sang-Youl Lee and Shi-Chang Wooh. Detection of stiffness reductions in laminated composite plates from their dynamic response using the microgenetic algorithm. Computational Mechanics, 36(4):320-330, April 2005.

[12] Scott W Doebling, Charles R Farrar, Michael B Prime, et al. A summary review of vibration-based damage identification methods. Shock and vibration digest, 30(2):91$105,1998$.

[13] John C. Brigham and Wilkins Aquino. Surrogate-Model Accelerated Random Search algorithm for global optimization with applications to inverse material identification. Computer Methods in Applied Mechanics and Engineering, 196(45-48):4561-4576, September 2007.

[14] Charles Hellier. Handbook of nondestructive evaluation. McGraw-Hill, 2001.

[15] JH Bungey. The validity of ultrasonic pulse velocity testing of in-place concrete for strength. NDT International, 13(6):296-300, 1980.

[16] M. J. W. Povey and J. M. Wilkinson. Application of ultrasonic pulse echo techniques to egg albumen quality testing: A preliminary report. British Poultry Science, 21(6):489495, 1980.

[17] D.C. Worlton. Ultrasonic Testing With Lamb Waves. Defense Technical Information Center, Sep 1956.

[18] R Jones. The ultrasonic testing of concrete. Ultrasonics, 1(2):78-82, 1963.

[19] Petro Moilanen. Ultrasonic guided waves in bone. IEEE transactions on ultrasonics, ferroelectrics, and frequency control, 55(6):1277-86, January 2008.

[20] Tom Pialucha and Peter Cawley. The detection of thin embedded layers using normal incidence ultrasound. Ultrasonics, 32(6):431-440, November 1994.

[21] T. Planès and E. Larose. A review of ultrasonic Coda Wave Interferometry in concrete. Cement and Concrete Research, 53:248-255, November 2013.

[22] Koen Van Den Abeele. Nonlinear Acoustic and Ultrasonic NDT of Aeronautical Components. AIP Conference Proceedings, 838:75-78, 2006.

[23] Bajram Zeqiri. Metrology for ultrasonic applications. Progress in biophysics and molecular biology, 93(1-3):138-52, 2007.

[24] Victor Giurgiutiu, Matthieu Gresil, Bin Lin, Adrian Cuc, Yanfeng Shen, and Catalin Roman. Predictive modeling of piezoelectric wafer active sensors interaction with highfrequency structural waves and vibration. Acta Mechanica, 223(8):1681-1691, March 2012. 
[25] Friedrich Moser, Laurence J Jacobs, and Jianmin Qu. Modeling elastic wave propagation in waveguides with the finite element method. Ndt $\mathscr{E}$ E International, 32(4):225-234, 1999.

[26] Joseph B Keller. Inverse Problems. The American Mathematical Monthly, 83(2):107118, 2014.

[27] Bahram Notghi and John C Brigham. Optimal nondestructive test design for maximum sensitivity and minimal redundancy for applications in material characterization. Smart Materials and Structures, 22(12):125036, December 2013.

[28] David G Luenberger. Optimization by vector space methods. John Wiley \& Sons, 1968.

[29] Hans-Paul Schwefel. Numerical optimization of computer models. John Wiley \& Sons, Inc., 1981.

[30] Samuel H. Brooks. A discussion of random methods for seeking maxima. Operations Research, 6(2):pp. 244-251, 1958.

[31] S.N. Deepa S.N. Sivanandam. Introduction to Genetic Algorithms. Springer Berlin Heidelberg, 2008.

[32] David Edward Goldberg et al. Genetic algorithms in search, optimization, and machine learning, volume 412. Addison-wesley Reading Menlo Park, 1989.

[33] Jung-Huai Chou and Jamshid Ghaboussi. Genetic algorithm in structural damage detection. Computers \&5 Structures, 79(14):1335-1353, 2001.

[34] M Azarbayejani, AI El-Osery, KK Choi, and MM Reda Taha. A probabilistic approach for optimal sensor allocation in structural health monitoring. Smart Materials and Structures, 17(5):055019, 2008.

[35] H Y Guo, L Zhang, L L Zhang, and J X Zhou. Optimal placement of sensors for structural health monitoring using improved genetic algorithms. Smart Materials and Structures, 13(3):528-534, June 2004.

[36] Daniel C. Kammer. Sensor set expansion for modal vibration testing. Mechanical Systems and Signal Processing, 19(4):700-713, July 2005.

[37] A Khan, D Ceglarek, and Jun Ni. Sensor location optimization for fault diagnosis in multi-fixture assembly systems. Journal of manufacturing science and engineering, 120(4):781-792, 1998.

[38] C. Papadimitriou. Optimal sensor placement methodology for parametric identification of structural systems. Journal of Sound and Vibration, 278(4-5):923-947, December 2004. 
[39] C. Papadimitriou, J. L. Beck, and S.-K. Au. Entropy-Based Optimal Sensor Location for Structural Model Updating. Journal of Vibration and Control, 6(5):781-800, January 2000 .

[40] AM Raich and TR Liszkai. Multi-bjective Optimization of Sensor and Excitation Layouts for Frequency Response Function-ased Structural Damage Identification. ComputerAided Civil and Infrastructure Engineering, 27:95-117, 2012.

[41] Gunwoo Noh and Klaus-Jürgen Bathe. An explicit time integration scheme for the analysis of wave propagations. Computers \& Structures, 129:178-193, 2013.

[42] H. Lewy R. Courant, K. Friedrrichs. Uber die partiellen differenzengl-eichungen der mathemtischen physik. Mathematische Annalen, 100:32-74, 1928.

[43] Thomas J. R. Hughes. The Finite Element Method. Prentice-Hall, 1987.

[44] FEniCS. http://fenicsproject.org/.

[45] SIMULIA. http://www.simulia.com/.

[46] MA Sumbatyan. A method of global random search in inverse problems with application to the problem of recognizing the shape of a defect. Journal of Applied Mathematics and Mechanics, 56(5):779-781, 1992.

[47] Charles Audet, Andrew J Booker, JE Dennis Jr, Paul D Frank, and Douglas W Moore. A surrogate-model-based method for constrained optimization. AIAA paper, 4891, 2000.

[48] B Yegnanarayana. Artificial neural networks. PHI Learning Pvt. Ltd., 2009.

[49] Carlos M Fonseca, Peter J Fleming, et al. Genetic algorithms for multiobjective optimization: Formulationdiscussion and generalization. In ICGA, volume 93, pages 416423, 1993. 